\title{
A Convenient Phosphine-Free Palladium-Catalyzed Direct Arylation of Thiazole under Mild Aerobic Conditions
}

Xiao-Xi He, Yan-Fang Li, Ju Huang*, Dong-Sheng Shen, Feng-Shou Liu*

School of Chemistry and Chemical Engineering, Guangdong Pharmaceutical University

Corresponding authors: Ju Huang, email: xianghuangj@163.com; Feng-Shou Liu, email: fengshou2004@126.com; 
Abstract: A series of bulky amine palladium complexes $\left\{\left[\left(\mathrm{Ar}-\mathrm{NH}_{2}\right)_{2} \mathrm{PdCl}_{2}\right]\right\}$ were synthesized and characterized. The catalytic activity of the palladium complexes was evaluated via the direct $\mathrm{C}-\mathrm{H}$ arylation of thiazoles with aryl bromides in aerobic conditions at $80-100{ }^{\circ} \mathrm{C}$. Under the optimal conditions, $0.5-0.05 \mathrm{~mol} \%$ of the bulky palladium complexes were found to be very efficient and produced the desired cross-coupling products in high yields.

Keywords: bulky aniline; palladium complex; thiazole; direct arylation; aerobic condition 


\section{Introduction}

With broad functional group tolerance and without the need for synthesis of organometallic coupling partners, palladium-catalyzed direct $\mathrm{C}-\mathrm{H}$ bond arylation has become an important method for the formation of carbon-carbon bonds during the past decade [1]. Particularly, the direct arylation of thiazoles has received much attention [2-4] because such structural units are frequently found in pharmaceuticals, natural products, and functional organic materials [5]. The efficiency of this cross-coupling is greatly influenced by the choice of ligand, base, solvent and additives [6]. The ligand, which binds to the palladium to stabilize the catalytic intermediates, plays a pivotal role in the transformation process. Thus, considerable efforts have been directed toward the development of efficient ligands [7]. Noticeable advances have been achieved with bulky and electron-rich phosphine ligands, which have significantly enlarged the scope of direct arylation [2g,h,n,o]. However, except some well-known air-stable phosphine developed by Buchwald, most of these phosphine-based ligands are not readily available, and they are air-sensitive and toxic [8].

In contrast, the recent development of phosphine-free and ligand-free catalysts has shown promise because they have the potential to overcome the disadvantages of catalytic instability and environmental concerns [7a-c]. Itami and coworkers were the first to report the palladium-catalyzed C4-selective arylation of thiazoles with arylboronic acids using 2,2'-bipyridyl (bipy) or 1,10-phenanthroline (phen) as the ligand [3h,r,s]. Subsequently, the $\mathrm{Pd} / \mathrm{bipy}$ 
was successfully applied for the C5-selective arylation of thiazole with aryl iodides [3g]. Murai and coworkers highlighted that commercial $\left[\mathrm{Pd}(\mathrm{phen})_{2}\right]\left(\mathrm{PF}_{6}\right)_{2}$ can promote the one-pot multiple direct arylation of thiazole with aryl halides in the presence of 5 mol\% catalyst loading [3f,i]. Recently, Doucet and Gök demonstrated the $N$-heterocyclic carbenes (NHCs) palladium catalysts have excellent performance in the preparation of arylthioazoles, even using the aryl bromides and chlorides as the coupling partners in a high reaction temperature $\left(>130^{\circ} \mathrm{C}\right)[3 \mathrm{e}, 3 \mathrm{p}]$. However, there are still much room to explore an efficient phosphine-free palladium catalyst for the direct $\mathrm{C}-\mathrm{H}$ arylation, especially with respect to the palladium loading $(<0.1 \mathrm{~mol} \%)$, the substrate scope of the method, and mild reaction temperature. Therefore, there is a need for readily available and efficient catalysts that can mediate the transformation under mild conditions.

Simple amines are highly efficient ligands for the Suzuki-Miyaura cross-coupling reaction, and they have a broad substrate scope [9]. Very recently, the use of dual $\mathrm{Pd} / \mathrm{Cu}$ catalysts in conjunction with amine ligands enabled the direct arylation of 2'-deoxyadenosines with both aryl iodides and bromides [10]. Moreover, Bulky substituted pyridine developed by Tsuji also exhibited highly efficient in air oxidation of alcohols [11]. Inspired by the promising performance of the nitrogen based ligands, we hypothesized that sterically bulky primary anilines, which can stabilize the palladium active species, would be beneficial for the direct arylation. Herein, we report a general 
method for the synthesis of 5-arylthiazoles using aniline palladium complexes under mildly aerobic reaction conditions.

\section{Results and discussion}

\subsection{Synthesis and characterization of aniline palladium complexes}

L2-L5 can be readily synthesized via the ortho-alkylation of anilines with diphenylmethanol in one-step, according to the procedure proposed by Markó and Nolan [12]. Moreover, the reaction of substituted anilines with palladium chloride in DMAc produced the corresponding C1-C5 in high yields (Scheme 1). These palladium complexes were characterized using NMR, MS, and elemental analysis. An advantage of these palladium complexes is that they are stable toward air and moisture, so that the preparation, purification and crystallization of the palladium complexes can be conveniently operated in open-air.

\section{<Insert Scheme 1>}

Crystals of C2-C5 that were suitable for X-ray diffraction were grown from a solution of dichloromethane and methanol in aerobic conditions. As shown in Figure 1-4, a slightly distorted square-planar coordination geometry was observed, in which the two aniline ligands are oriented trans to each other in the C2-5. The nitrogens, $\mathrm{N}(1)$ and $\mathrm{N}(2)$, and chlorines, $\mathrm{Cl}(1)$ and $\mathrm{Cl}(2)$ that are attached to palladium are all in the same plane. The solid state structure of these four palladium complexes revealed similar bond lengths. Nevertheless, it is deserved to note that the Pd-N bond lengths 
(2.056(3) $\AA$ ) in $\mathbf{C} 2$ were shorter than that of the typical $\mathrm{Pd}-\mathrm{N}\left(\mathrm{sp}^{3}\right)$ single bond length (2.07-2.10 $\mathrm{A})$ and that of C2-5, indicating a slightly stronger interaction between the coordinated atoms and the palladium in $\mathbf{C 2}[9 \mathrm{~b}, \mathrm{i}, 13]$.

\section{<Insert Figure 1> \\ $<$ Insert Figure 2> \\ <Insert Figure 3> \\ <Insert Figure 4>}

\subsection{Direct arylation catalyzed by aniline palladium complexes}

Initially, we chose 4-methylthiazole (1a) and 4-bromobenzonitrile (2a) as the model substrates in the presence of $0.5 \mathrm{~mol} \%$ of the palladium complex (Table 1). To establish mild reaction conditions, the heteroarene and the aryl bromides were employed in equimolar quantities. The experiments were performed at a temperature of $80{ }^{\circ} \mathrm{C}$ for $24 \mathrm{~h}$, using $\mathrm{K}_{2} \mathrm{CO}_{3}$ as the base, DMAc as the solvent, and pivalic acid (PivOH) as the additive. As shown in Table 1, the precatalysts critically affected the efficiency of this reaction. $\mathrm{Cy}_{2} \mathrm{NH} / \mathrm{PdCl}_{2}$, which was proved highly efficient in the Suzuki-Miyaura coupling [9a, b], showed promising results, and it provided the direct arylation product, 3aa, in a 86\% GC yield (Entry 1, Table 1). Meanwhile, C1, with 2,6-diisopropyl substituents on the aniline moiety, provided the coupling product, 3aa, with almost the same efficiency as the $\mathrm{Cy}_{2} \mathrm{NH} / \mathrm{PdCl}_{2}$. In contrast, the bulky benzhydryl group on the aniline moiety exhibited a profound effect on the activities. For example, C2-C5 exhibited superior reactivity than $\mathbf{C 1}$ and 
$\mathrm{Cy}_{2} \mathrm{NH} / \mathrm{PdCl}_{2}$ (Entries 3-6, Table 1). Reasonably, the use of bulky steric of the ligand, which can stabilize the palladium center and further facilitate the elimination rate, is essential for the direct arylation process [2i, 14]. However, quite out of our expectation, in these aniline palladium complexes screened, C2, which is less bulky than C3-C5, was found to be the most active catalyst in the direct arylation. For example, the coupling reaction that employed $\mathbf{C 2}$ as the precatalyst afforded the corresponding product, 3aa, in an almost quantifiable conversion, and an isolated yield of $92 \%$ was obtained (Entry 3, Table 1). Probably, bearing the 2,6-benzhydryl group on aniline moiety, the most bulky precatalysts C3-C5 was too crowed. In contrast, with mono benzhydryl group on C2, it was sufficient for stabilizing the palladium center and the less bulky steric would facilitate the rate of oxidative addition process.

\section{$\langle$ Insert Table 1>}

Based on the above promising results, other reaction conditions for the direct $\mathrm{C}-\mathrm{H}$ arylation were then investigated. As shown in Table 2, the base, solvent, and acid additives were all crucial to the efficiency of this reaction. Among the bases such as $\mathrm{K}_{2} \mathrm{CO}_{3}, \mathrm{Na}_{2} \mathrm{CO}_{3}, \mathrm{NaHCO}_{3}, \mathrm{C}_{6} \mathrm{H}_{5} \mathrm{CO}_{2} \mathrm{Na}, \mathrm{NaOAc}$, $\mathrm{K}_{3} \mathrm{PO}_{4}$ and KOAc examined, the use of $\mathrm{K}_{2} \mathrm{CO}_{3}$ as a base led to best yield (Entries 1-7, Table 2). However, the bases, such as $\mathrm{NaOH}$ and $\mathrm{Cs}_{2} \mathrm{CO}_{3}$, were much less effective and produced a minor yield of $6 \%$ and $5 \%$, respectively (Entries 8 and 9, Table 2). Furthermore, no products were observed when $\mathrm{KOH}$, KOtBu and $\mathrm{NaOtBu}$, were used (Entries 10-12, Table 2). A number of solvents 
were then screened, and the solvents, such as DMF, dioxane, toluene, xylene and N-methyl-2-pyrrolidone (NMP), all led to the formation of the product in moderate to good yields (Entries 13-17, Table 2), whereas DMAc produced the best yield (Entry 1, Table 2). The acid additives were also evaluated and it was found that only a $9 \%$ product yield was observed when there was no acid additive under the standard conditions (Entry 20, Table 2). In contrast, there was a significant acceleration effect when introducing the carboxylic acid additive to the reaction. For example, acetic acid and propanoic acid produce moderate yields of 65 and 64\%, respectively (Entries 21-22, Table 2). Moreover, among the screened acid additives, PivOH provided the best yield for the coupling reaction product (Entry1, Table 2). The indispensable of the acid additives is in accordance with a concerted metalation-deprotonation (CMD) pathway [15]. Thus, the optimization results were obtained by using a mixture of thiazole (1.0 equiv), aryl bromide (1.0 equiv), $\mathbf{C 2}$ as the precatalyst, $\mathrm{K}_{2} \mathrm{CO}_{3}$ as the base, and $\mathrm{PivOH}$ as the acid additive in DMAc. Under our reaction conditions, only a trace amount of side products, such as homocoupling biaryl products that were derived from the aryl bromides and C-2 arylation of the thiazoles, were observed during the course of this reaction.

\section{<Insert Table 2>}

After the reaction conditions were optimized, the scope of the direct arylation between a variety of aryl bromides and 4-methylthiazole was explored. As shown in Table 3, in the presence of $0.5 \mathrm{~mol} \%$ of $\mathbf{C 2}$, the cross-coupling 
reagents smoothly reacted to afford the desired products at $80{ }^{\circ} \mathrm{C}$. Moreover, a wide range of functional groups on the aryl bromides were tolerated well during this process, allowing further transformations or modifications. For example, the electron-deficient aryl bromides, such as 4-bromobenzonitrile, 1-bromo-4-chlorobenzene, 4-bromobenzaldehyde, and methyl 4-bromobenzoate, provided the corresponding products, 3aa-3ac and 3aj, in excellent yields (86-94\%). The electron-deficient substrates bearing the fluoroand trifluoro-methyl heteroatoms, which are important motifs in pharmaceuticals, were produced in high yields (76-97\%). Additionally, some electron-deficient substrate partners, which bear the nitro $\left(-\mathrm{NO}_{2}\right)$ and acetyl (-COMe) groups, commonly produced low yields in previous reports. ${ }^{2 \mathrm{k}}$ It provided the corresponding products, 3ad and 3ai, in high yields of $88 \%$. Regarding the electron-neutral and electron-rich aryl bromides, such as 1-bromobenzene, 4-bromotoluene, 3-bromotoluene, 3-bromoanisole, and 1-bromonaphthalene, they were also well tolerated and yielded the biaryl products, 3al, 3am, 3an, 3ap and 3ar, in satisfactory yields (55-92\%). However, the sterically hindered substrates and less active substrates, such as 1-bromo-2-methylbenzene and 4- bromoanisole, produced 3ao and 3aq in much lower yields of $31 \%$ and $39 \%$, respectively.

\section{$<$ Insert Table 3>}

Decreasing the amount of the palladium to catalyze the reaction process is desirable not only because of cost, but also in order to facilitate metal removal 
once the reaction is complete. The outstanding performance of $\mathbf{C 2}$ naturally led us to examine the effect of reducing the catalyst loading. Remarkably, both the electron-deficient and electron-rich aryl bromides were easily reacted with thiazole to produce the corresponding products using a lower palladium loading of $0.1 \mathrm{~mol} \%$ in moderate to excellent yields, if the temperature was raised to $100^{\circ} \mathrm{C}$ (Table 4). When the amount of catalyst was further reduced to 0.05 mmol\%, the electron-deficient aryl bromides, such as 1-bromo-4-chlorobenzene, 4-bromobenzonitrile, and 4-bromobenzaldehyde, also can provide the corresponding products (3aa-3ab and 3ac) in moderate to high yields (71-82\%). Moreover, a highly efficient direct C5-arylation was also achieved when 2,4-dimethylthiazole was introduced. The methyl group on the C-2 position of the thiazole did not cause obvious steric inhibition, and it provided the desired products, $\mathbf{3 b b}-\mathbf{3 b l}$, in good to excellent yields.

\section{<Insert Table 4>}

By probing the aggregation state of the catalytic species and the mechanism of the palladium-catalyzed direct arylation, important insight into the $\mathrm{C}-\mathrm{H}$ bond functionality can be provided. Generally, the transformations via ligand-based catalytic systems have been recognized as homogenous catalytic processes [16]. In these cases, the ligands, which could stabilize the palladium, played a critical role in the catalytic efficiency and regioselectivity under mild reaction conditions. However, the ligand-free palladium catalysts could also successfully achieve the direct arylation under carefully controlled conditions, 
such as at elevated temperature $\left(>130{ }^{\circ} \mathrm{C}\right)$ and in polar solvent [3d,17]. In the latter case, the heterogeneous palladium nanoparticles (PdNPs) were generated in situ, and the cross-coupling reaction occurred via the interaction of the substrates with the palladium on the outer rim of the nanoparticles [18]. Nevertheless, the species involved in the palladium-catalyzed cross-coupling is still not clear, and the possible aggregation states of the palladium require further research. Very recently, Fairlamb et al. demonstrated that in the piperidine-activated palladium/copper direct arylation, the monodentate nitrogen atoms are prone to decoordination from the palladium center even when a homogenous palladium precursor was used [10c]. Moreover, the transmission electron microscopy (TEM) suggested that the PdNPs were generated under a mild reaction temperature of $80^{\circ} \mathrm{C}$.

To determine if the PdNPs are involved in the catalytic cycle, we first performed poisoning experiments with the aniline-based palladium precatalyst. Quantitative poisons, such as $\mathrm{PPh}_{3}$ and $\mathrm{CS}_{2}(<1$ equiv relative to the palladium loading), can effectively terminate the reaction activity, thus confirming the heterogeneous nature of the $\operatorname{Pd}(0)$ catalyst in the cross-coupling reaction[19]. Considering the low boiling point of $\mathrm{CS}_{2}$, we first checked the $\mathrm{PPh}_{3}$ poisoning method. The reaction was performed using 4-bromobenzonitrile (1 mmol), 4-methylthiazole $(1 \mathrm{mmol}), \mathrm{K}_{2} \mathrm{CO}_{3}(1.5 \mathrm{mmol}), \mathrm{PivOH}(0.3 \mathrm{mmol})$, and $\mathbf{C 2}$ (0.001 mmol) in DMAc (3 mL) at $100{ }^{\circ} \mathrm{C}$ for $24 \mathrm{~h}$. An excess (100 equiv relative to the palladium) of $\mathrm{PPh}_{3}$ was added at the beginning of the reaction, 
and the catalytic activity of $\mathbf{C 2}$ showed a high GC yield of $86 \%$, indicating a negative effect. Moreover, a $\operatorname{Hg}(0)$ poison test (300 equiv relative to the palladium) was also conducted, and the catalytic activity was not quenched, but it produced a lower GC yield of $48 \%$. Furthermore, submitting the samples of the reaction mixtures to TEM imaging after the reaction did not produce any aggregated nanoparticles, indicating the homogenous nature of the catalytic system. Thus, we conclude that the excellent catalytic performance of the aniline palladium catalyst is attributed to the ligand structure.

In order to obtain further information on the mechanism of the cross-coupling, we conducted the stoichiometric reaction of $\mathrm{PivOH} / \mathrm{K}_{2} \mathrm{CO}_{3}$ with precatalyst $\mathbf{C} 2$ in DMAc solution at a temperature of $80^{\circ} \mathrm{C}$. The preliminary investigation involved tracing the catalytic species with ESI-MS (seen in Figure S6). The result showed a peak at $\mathrm{m} / \mathrm{z}=570.1$, which could be formed from $[\mathrm{L} 2 \mathrm{Pd}(\mathrm{PivO}) \mathrm{Cl}] \mathrm{K}^{+}$. Moreover, the ${ }^{13} \mathrm{C}$ NMR analysis of the obtained compound showed a resonance at 161.7 and 160.6 ppm, which suggested the ligand exchange between halide with the pivalic anion (seen in Figure S7). Subsequently, we investigated the direct arylation between 4-bromobenzonitrile and 4-methylthiazole in the presence of a $10 \mathrm{~mol} \%$ amount of $\mathrm{C2}$ at $80{ }^{\circ} \mathrm{C}$ for $2 \mathrm{~h}$. The reaction proceeded in $52 \%$ GC yield and the mixture was determined by ESI-MS (seen in Figure S8). It was found the peaks at $\mathrm{m} / \mathrm{z}=963.7$ and 696.6, which could be formed from [2 $\left.\mathrm{L} 2 \mathrm{Pd}(4-m e t h y l t h i a z o l e)\left(\mathrm{C}_{6} \mathrm{H}_{4} \mathrm{CN}\right) \mathrm{Br}\right]^{+}$and $[$ L1Pd(4-methylthiazole $\left.)\left(\mathrm{C}_{6} \mathrm{H}_{4} \mathrm{CN}\right)(\mathrm{PivO})\right]^{+}$. These result suggested that an oxidative addition of $\operatorname{Pd}(0)$ with 4-bromobenzonitrile and subsequently a smooth ligand 
exchange would be involved in the process of the direct arylation.

On the basis of the above observations, we propose plausible catalytic cycles [20]. As can be seen in Scheme 2, when aniline palladium complex was used, the reduction of $\mathrm{Pd}(\mathrm{II})$ to $\mathrm{Pd}(0)$ (A) would be taken in situ. Then, the oxidative of aryl bromide with $\mathrm{Pd}(0)$ to afford the aryl Pd(II) species (B). Subsequently, an exchange of the bromide ligand with pivalic anion allowed for a related CMD type transition state (C) to afford the diaryl-Pd(II) intermediate (D). After the reductive elimination rendering the target arylated product, the release $\operatorname{Pd}(0)$ species can enter the next catalytic cycle.

\section{Conclusions}

We developed a class of aniline palladium complexes for the direct arylation of thiazoles by incorporating the bulky ortho- $\mathrm{CH}_{2} \mathrm{Ph}_{2}$ framework. These aniline ligands, which contain a protected palladium center, have provided a highly versatile strategy for direct $\mathrm{C}-\mathrm{H}$ bond functionality reactions. In addition to a broad substrate scope, including electron-rich and deficient aryl bromides, the simple precatalyst, C2, also demonstrated its catalytic ability under mild reaction conditions for low palladium loading. Our investigation into the mechanism revealed that a homogenous process is involved in the cross-coupling reaction.

\section{Experimental}

4.1 Physical Measurements and Materials 
2,6-Diisopropylaniline was purchased from Aldrich Chemical and were distilled under reduced pressure before being used. Palladium chloride was purchased from Aldrich Chemical. 2,6-diphenylmethyl-4-methylaniline, 2,6-diphenylmethyl-4-chloroaniline and 2,6-diphenylmethyl-4-methoxylaniline, were prepared according to literature methods [12]. The NMR data of compounds were obtained on a Varian Mercury-Plus $400 \mathrm{MHz}$ spectrometer at ambient temperature with the decoupled nucleus, using $\mathrm{CDCl}_{3}$ as solvent and referenced versus TMS as standard. Elemental analyses were determined with a Vario EL Series Elemental Analyzer from Elementar. The X-ray diffraction data of single crystals were obtained with the $\omega-2 \theta$ scan mode on a Bruker SMART 1000 CCD diffractiometer with graphite-monochromated Mo $\mathrm{K} \alpha$ radiation $(\lambda=0.71073 \AA)$ at $173 \mathrm{~K}$ for $\mathbf{C 2}, \mathbf{C 3}$ and $\mathbf{C 4}, \mathrm{Cu} \mathrm{K} \alpha$ radiation $(\lambda=1.54178 \AA)$ at $173 \mathrm{~K}$ for $\mathbf{C 5}$. The structure was solved using direct methods, and further refinement with full-matrix least squares on $F^{2}$ was obtained with the SHELXTL program package. All non-hydrogen atoms were refined anisotropically. Hydrogen atoms were introduced in calculated positions with the displacement factors of the host carbon atoms. CCDC 1401361-1401364 (C2-C5) contain the supplementary crystallographic data for this paper.

\subsection{General procedures for the synthesis of aniline palladium compounds}

Aniline ligand $(10.0 \mathrm{mmol})$ and palladium dichloride $(0.886 \mathrm{~g}, 5.0 \mathrm{mmol})$ were dissolved in $15 \mathrm{~mL}$ of DMAc at room temperature. After the mixture was stirred for $0.5 \mathrm{~h}$ at $80{ }^{\circ} \mathrm{C}$, the methanol $(50 \mathrm{~mL})$ was added and the precipitation was formed. The precipitate of palladium complexes was then dissolved in $5 \mathrm{~mL}$ dichloromethane, 
then $20 \mathrm{~mL}$ hexane was added. After crystallized from the mixture of ethanol and dichloromethylene, the palladium complex was obtained as light yellow crystals.

\subsubsection{Synthesis of [2,6-(iPr $\left.)_{2} \mathrm{C}_{6} \mathrm{H}_{3} \mathrm{NH}\right]_{2} \mathrm{PdCl}_{2}(\mathrm{Cl})$}

${ }^{1} \mathrm{H}$ NMR $\left(400 \mathrm{MHz}, \mathrm{CDCl}_{3}\right): \delta(\mathrm{ppm}) 7.23\left(\mathrm{t}, \mathrm{J}_{\mathrm{HH}}=7.6 \mathrm{~Hz}, \mathrm{Ar}-\mathrm{H}, 2 \mathrm{H}\right), 7.12\left(\mathrm{t}, \mathrm{J}_{\mathrm{HH}}=7.6\right.$ $\mathrm{Hz}, \mathrm{Ar}-\mathrm{H}, 4 \mathrm{H}), 4.69$ (s, NH $\left.\mathrm{H}_{2}, 4 \mathrm{H}\right), 3.57\left(\mathrm{~m}, \mathrm{CH}\left(\mathrm{CH}_{3}\right)_{2}, 4 \mathrm{H}\right), 1.36\left(\mathrm{~m}, \mathrm{CH}\left(\mathrm{CH}_{3}\right)_{2}, 24 \mathrm{H}\right)$.

${ }^{13} \mathrm{C}$ NMR (100 MHz, $\left.\mathrm{CDCl}_{3}\right): \delta$ (ppm) 139.8, 133.4, 126.5, 123.6, 28.2, 23.5. ESI-MS. $m / z: 497,\left[2 \mathrm{~L}_{1} \mathrm{PdCl}\right]^{+} ; 461,\left[2 \mathrm{~L}_{2} \mathrm{Pd}\right]^{2+}$; Anal. Calcd for: $\mathrm{C}_{24} \mathrm{H}_{38} \mathrm{Cl}_{2} \mathrm{~N}_{2} \mathrm{Pd}: \mathrm{C}, 54.19 ; \mathrm{H}$, 7.20; N, 5.27. Found: C, 54.01; H, 7.28; N, 5.16. $\mathrm{T}_{\mathrm{m}}$ : $230.5^{\circ} \mathrm{C}$.

\subsubsection{Synthesis of [2-( $\left.\left.\mathrm{CHPh}_{2}\right)-4,6-\left(\mathrm{CH}_{3}\right)_{2} \mathrm{C}_{6} \mathrm{H}_{2} \mathrm{NH}\right]_{2} \mathrm{PdCl}_{2}(\mathrm{C2})$}

${ }^{1} \mathrm{H}$ NMR $\left(400 \mathrm{MHz}, \mathrm{CDCl}_{3}\right): \delta(\mathrm{ppm}) 7.32$ (m, Ar-H, 20H), $6.80(\mathrm{~s}, \mathrm{Ar}-\mathrm{H}, 2 \mathrm{H}), 6.80$ (s, Ar-H, 2H), $6.48(\mathrm{~s}, \mathrm{Ar}-\mathrm{H}, 2 \mathrm{H}), 6.37\left(\mathrm{~s}, \mathrm{CHPh}_{2}, 2 \mathrm{H}\right), 4.17$ (s, NH$\left.H_{2}, 4 \mathrm{H}\right), 2.44$ (s, $\left.\mathrm{CH}_{3}, 6 \mathrm{H}\right), 2.08\left(\mathrm{~s}, \mathrm{CH}_{3}, 6 \mathrm{H}\right) .{ }^{13} \mathrm{C}$ NMR $\left(100 \mathrm{MHz}, \mathrm{CDCl}_{3}\right): \delta(\mathrm{ppm})$ 141.6, 135.8, 135.1, 133.8, 130.1, 129.8, 129.2, 128.9, 128.7, 127.0, 51.6, 21.2, 18.9. ESI-MS. $m / z$ : 715, $\left[2 \mathrm{~L}_{2} \mathrm{PdCl}\right]^{+} ; 681,\left[2 \mathrm{~L}_{2} \mathrm{Pd}\right]^{2+} ; 288,\left[\mathrm{~L}_{2}\right]^{+}$. Anal. Calcd for: $\mathrm{C}_{42} \mathrm{H}_{42} \mathrm{Cl}_{2} \mathrm{~N}_{2} \mathrm{Pd}: \mathrm{C}$, 67.07; H, 5.63; N, 3.72. Found: C, 66.78; H, 5.71; $\mathrm{N}, 3.65 . \mathrm{T}_{\mathrm{m}}: 248.0{ }^{\circ} \mathrm{C}$.

\subsubsection{Synthesis of [2,6-(CHPh$\left.)_{2}-4-\left(\mathrm{CH}_{3}\right) \mathrm{C}_{6} \mathrm{H}_{2} \mathrm{NH}\right]_{2} \mathrm{PdCl}_{2}(\mathrm{C3})$}

${ }^{1} \mathrm{H}$ NMR (400 MHz, CDCl 3 ): $\delta(\mathrm{ppm}) 7.24$ (m, Ar-H, 40H), 6.45 (s, Ar-H, 4H), 6.44 (s, $\left.\mathrm{CHPh}_{2}, 4 \mathrm{H}\right), 4.11\left(\mathrm{~s}, \mathrm{NH}_{2}, 4 \mathrm{H}\right), 2.03\left(\mathrm{~s}, \mathrm{CH}_{3}, 6 \mathrm{H}\right) .{ }^{13} \mathrm{C} \mathrm{NMR}\left(100 \mathrm{MHz}, \mathrm{CDCl}_{3}\right): \delta$ (ppm) 141.4, 136.1, 134.9, 133.6, 129.7, 129.6, 128.6, 127.1, 51.7, 21.5. ESI-MS. $m / z$ : 584, $\left[\mathrm{L}_{3} \mathrm{PdCl}\right]^{+} ; 440,\left[\mathrm{~L}_{3}\right]^{+}$. Anal. Calcd for: $\mathrm{C}_{66} \mathrm{H}_{58} \mathrm{Cl}_{2} \mathrm{~N}_{2} \mathrm{Pd}: \mathrm{C}, 75.03 ; \mathrm{H}, 5.53 ; \mathrm{N}$, 2.65. Found: C, $74.96 ; \mathrm{H}, 5.60 ; \mathrm{N}, 2.61 . \mathrm{T}_{\mathrm{m}}: 280.6{ }^{\circ} \mathrm{C}$. 
${ }^{1} \mathrm{H}$ NMR (400 MHz, $\left.\mathrm{CDCl}_{3}\right): \delta(\mathrm{ppm}) 7.24$ (m, Ar-H, 40H), 6.45 (s, Ar-H, 4H), 6.19 (s, $\left.\mathrm{CHPh}_{2}, 4 \mathrm{H}\right), 4.04\left(\mathrm{~s}, \mathrm{NH}_{2}, 4 \mathrm{H}\right), 3.42\left(\mathrm{~s}, \mathrm{CH}_{3}, 6 \mathrm{H}\right) .{ }^{13} \mathrm{C} \mathrm{NMR}\left(100 \mathrm{MHz}, \mathrm{CDCl}_{3}\right): \delta$ (ppm) 156.6, 141.1, 137.8, 129.7, 129.2, 128.7, 127.2, 114.5, 54.8, 51.8. ESI-MS. $m / z$ : 456, $\left[\mathrm{L}_{4}\right]^{+}$. Anal. Calcd for: $\mathrm{C}_{66} \mathrm{H}_{58} \mathrm{Cl}_{2} \mathrm{~N}_{2} \mathrm{O}_{2} \mathrm{Pd}: \mathrm{C}, 72.83 ; \mathrm{H}, 5.37 ; \mathrm{N}, 2.57$. Found: $\mathrm{C}$, 72.67; H, 5.41; N, 2.51. $\mathrm{T}_{\mathrm{m}}: 282.4^{\circ} \mathrm{C}$.

\subsubsection{Synthesis of $\left[2,6-\left(\mathrm{CHPh}_{2}\right)_{2}-4-(\mathrm{Cl}) \mathrm{C}_{6} \mathrm{H}_{2} \mathrm{NH}\right]_{2} \mathrm{PdCl}_{2}$ (C5)}

${ }^{1} \mathrm{H}$ NMR (400 MHz, CDCl $): \delta(\mathrm{ppm}) 7.29$ (m, Ar-H, 7H), 7.25 (m, Ar-H, 33H), 6.63 (s, Ar-H, 4H), $6.40\left(\mathrm{~s}, \mathrm{CHPh}_{2}, 4 \mathrm{H}\right), 4.15\left(\mathrm{~s}, \mathrm{NH} \mathrm{H}_{2}, 4 \mathrm{H}\right) .{ }^{13} \mathrm{C} \mathrm{NMR}\left(100 \mathrm{MHz}, \mathrm{CDCl}_{3}\right)$ : $\delta(\mathrm{ppm}) 140.4,138.3,134.9,131.4,129.6,129.0,128.9,127.6,51.8$. ESI-MS. $m / z$ : 460, $\left[\mathrm{L}_{5}\right]^{+}$. Anal. Calcd for: $\mathrm{C}_{64} \mathrm{H}_{52} \mathrm{Cl}_{4} \mathrm{~N}_{2} \mathrm{Pd}: \mathrm{C}, 70.05 ; \mathrm{H}, 4.78 ; \mathrm{N}, 2.55$. Found: C, 69.88; H, 4.86; N, 2.51. $\mathrm{T}_{\mathrm{m}}: 283.2{ }^{\circ} \mathrm{C}$.

\subsection{General Procedure for Direct Arylation promoted by palladium complexes}

Unless otherwise noted, the direct arylation reactions were carried out under aerobic conditions. Reaction temperatures are reported as the temperature of the heating the vessel unless otherwise stated. All solvents were not purified as received. In the parallel reaction instrument containing tubes were charged with palladium complexes, aryl bromide $(1.0 \mathrm{mmol})$, heteroarene $(1.0 \mathrm{mmol}), \mathrm{K}_{2} \mathrm{CO}_{3}(1.5 \mathrm{mmol})$, PivOH $(0.3 \mathrm{mmol})$ and $3 \mathrm{~mL}$ of DMAc. The reaction mixture was carried out at 100 ${ }^{\circ} \mathrm{C}$ for $24 \mathrm{~h}$. After completion of the reaction, the reaction mixture was cooled to room temperature and $20 \mathrm{~mL}$ of water was added. The mixture was diluted with $\mathrm{Et}_{2} \mathrm{O}$ (5 $\mathrm{mL})$, followed by extraction three times $(3 \times 5 \mathrm{~mL})$ with $\mathrm{Et}_{2} \mathrm{O}$. The organic layer was dried with anhydrous $\mathrm{MgSO}_{4}$, filtered and evaporated under vacuum. The crude 
products were purified by silica-gel column chromatography using petroleum ether-ethyl acetate (20/1, V/V) as an eluent, and the isolated yield was then calculated based on the feeding of the aryl halide. The isolated corresponding products were characterized by ${ }^{1} \mathrm{H}$ NMR and ${ }^{13} \mathrm{C}$ NMR, and the spectrums are reported in Supporting Information.

\subsubsection{4-(4-methylthiazol-5-yl)benzonitrile 3aa [3d]}

${ }^{1} \mathrm{H}$ NMR (400 MHz, $\mathrm{CDCl}_{3}$ ), $\delta(\mathrm{ppm}): 8.76$ (s, Ar-H, 1H), 7.74 (d, J=8.7 Hz, Ar-H, 2H), $7.58(\mathrm{~d}, \mathrm{~J}=8.7 \mathrm{~Hz}, \mathrm{Ar}-\mathrm{H}, 2 \mathrm{H}), 2.58\left(\mathrm{~s}, \mathrm{CH}_{3}, 3 \mathrm{H}\right) .{ }^{13} \mathrm{C} \mathrm{NMR}\left(100 \mathrm{MHz}, \mathrm{CDCl}_{3}\right), \delta$ (ppm): 151.5, 150.0, 136.8, 132.5, 130.0, 129.7, 118.4, 111.5, 16.3.

\subsubsection{5-(4-chlorophenyl)-4-methylthiazole 3ab [2k]}

${ }^{1} \mathrm{H}$ NMR (400 MHz, $\mathrm{CDCl}_{3}$ ), $\delta$ (ppm): 8.69 (s, Ar-H, 1H), 7.42 (m, Ar-H, 4H), 2.53 (s, $\left.\mathrm{CH}_{3}, 3 \mathrm{H}\right) .{ }^{13} \mathrm{C} \mathrm{NMR}\left(100 \mathrm{MHz}, \mathrm{CDCl}_{3}\right), \delta(\mathrm{ppm}): 150.4,148.8,133.9,130.6,130.4$, $130.4,129.1,16.0$

\subsubsection{4-(4-methylthiazol-5-yl)benzaldehyde3ac [2r]}

${ }^{1} \mathrm{H}$ NMR (400 MHz, CDCl 3 ), $\delta$ (ppm): 10.03 (s, CHO, 1H), 8.74 (s, Ar-H, 1H), 7.94 (m, Ar-H, 2H), 7.62 (m, Ar-H, 2H), $2.58\left(\mathrm{~s}, \mathrm{CH}_{3}, 3 \mathrm{H}\right) .{ }^{13} \mathrm{C}$ NMR (100 MHz, $\left.\mathrm{CDCl}_{3}\right)$, $\delta$ (ppm): 191.4, 151.4, 149.8, 138.2, 135.3, 130.7, 130.0, 129.6, 16.4.

\subsubsection{4-methyl-5-(4-nitrophenyl)thiazole 3ad [3m]}

${ }^{1} \mathrm{H}$ NMR (400 MHz, CDCl 3 ): $\delta(\mathrm{ppm}) 8.78(\mathrm{~s}, \mathrm{Ar}-\mathrm{H}, 1 \mathrm{H}), 8.30(\mathrm{~m}, \mathrm{Ar}-\mathrm{H}, 2 \mathrm{H}), 7.63$ (m, Ar-H, 2H), $2.59\left(\mathrm{~s}, \mathrm{CH}_{3}, 3 \mathrm{H}\right) .{ }^{13} \mathrm{C}$ NMR (100 MHz, $\left.\mathrm{CDCl}_{3}\right): \delta(\mathrm{ppm}) 151.8,150.4$, $147.0,138.8,129.9,129.8,124.0,16.4$ 
${ }^{1} \mathrm{H}$ NMR (400 MHz, $\mathrm{CDCl}_{3}$ ): $\delta(\mathrm{ppm}) 8.77$ (s, Ar-H, 1H), 7.38 (m, Ar-H, 2H), 7.21 (m, Ar-H, 2H), $2.43\left(\mathrm{~s}, \mathrm{CH}_{3}, 3 \mathrm{H}\right) .{ }^{13} \mathrm{C} \mathrm{NMR}\left(100 \mathrm{MHz}, \mathrm{CDCl}_{3}\right): \delta$ (ppm) 160.9, 158.4, 151.7, 150.8, 132.1, 130.2 (d, J=8.2 Hz), 124.2 (d, J=3.7 Hz), 119.4 (d, J=15.2 Hz), 116.1(d, J=21.9 Hz), $15.9(\mathrm{~d}, \mathrm{~J}=2.8 \mathrm{~Hz})$.

\subsubsection{5-(3-fluorophenyl)-4-methylthiazole 3af [3m]}

${ }^{1} \mathrm{H}$ NMR (400 MHz, $\mathrm{CDCl}_{3}$ ): $\delta$ (ppm) 8.71 (s, Ar-H, 1H), 7.41 (m, Ar-H, 1H), 7.22 (m, Ar-H, 1H), $7.16(\mathrm{~m}, \mathrm{Ar}-\mathrm{H}, 1 \mathrm{H}), 7.08(\mathrm{~m}, \mathrm{Ar}-\mathrm{H}, 1 \mathrm{H}), 2.54\left(\mathrm{~s}, \mathrm{CH}_{3}, 3 \mathrm{H}\right) .{ }^{13} \mathrm{C} \mathrm{NMR}$ (100 MHz, $\left.\mathrm{CDCl}_{3}\right): \delta(\mathrm{ppm}) 163.9,161.4,150.7,149.1,134.0(\mathrm{~d}, \mathrm{~J}=8.2 \mathrm{~Hz}), 130.2(\mathrm{~d}$, $\mathrm{J}=8.5 \mathrm{~Hz}), 125.0$ (d, J=2.9 Hz), 116.1 (d, J=12.2 Hz), 114.8 (d, J=20.9 Hz), 16.1.

\subsubsection{5-(4-fluorophenyl)-4-methylthiazole 3ag [3m]}

${ }^{1} \mathrm{H}$ NMR $\left(400 \mathrm{MHz}, \mathrm{CDCl}_{3}\right): \delta(\mathrm{ppm}) 8.67(\mathrm{~s}, \mathrm{Ar}-\mathrm{H}, 1 \mathrm{H}), 7.41(\mathrm{t}, \mathrm{J}=7.2 \mathrm{~Hz}, \mathrm{Ar}-\mathrm{H}$, 2H), 7.13 (m, J=8.0 Hz, Ar-H, 2H), $2.50\left(\mathrm{~s}, \mathrm{CH}_{3}, 3 \mathrm{H}\right) .{ }^{13} \mathrm{C} \mathrm{NMR}\left(100 \mathrm{MHz}, \mathrm{CDCl}_{3}\right)$ : $\delta(\mathrm{ppm})$ 163.6, 161.1, 150.3, 148.5, 131.0 (d, J=8.1 Hz), 127.8 (d, J=3.4 Hz), 115.7 (d, $\mathrm{J}=21.6 \mathrm{~Hz}), 15.9$.

\subsubsection{5-(3,5-bis(trifluoromethyl)phenyl)-4-methylthiazole 3ah [21]}

${ }^{1} \mathrm{H}$ NMR (400 MHz, $\left.\mathrm{CDCl}_{3}\right): \delta(\mathrm{ppm}) 8.78(\mathrm{~s}, \mathrm{Ar}-\mathrm{H}, 1 \mathrm{H}), 7.87$ (s, Ar-H, 3H) $2.55(\mathrm{~s}$, $\left.\mathrm{CH}_{3}, 3 \mathrm{H}\right) .{ }^{13} \mathrm{C} \mathrm{NMR}\left(100 \mathrm{MHz}, \mathrm{CDCl}_{3}\right): \delta(\mathrm{ppm}) 151.7,150.4,134.3,132.2(\mathrm{q}$, $\mathrm{J}=33.4 \mathrm{~Hz}), 129.2,128.7,123.0(\mathrm{q}, \mathrm{J}=271.2 \mathrm{~Hz}), 121.6(\mathrm{q}, \mathrm{J}=3.7 \mathrm{~Hz}), 16.0$.

\subsubsection{1-(4-(4-methylthiazol-5-yl)phenyl)ethanone 3ai [2x]}

${ }^{1} \mathrm{H}$ NMR (400 MHz, $\mathrm{CDCl}_{3}$ ): $\delta$ (ppm) 8.66 (m, Ar-H, 1H), 7.94 (m, Ar-H, 2H), 7.48 (m, Ar-H, 2H), $2.56\left(\mathrm{~m}, \mathrm{CH}_{3}, 3 \mathrm{H}\right), 2.49\left(\mathrm{~m}, \mathrm{CH}_{3}, 3 \mathrm{H}\right) .{ }^{13} \mathrm{C} \mathrm{NMR}\left(100 \mathrm{MHz}, \mathrm{CDCl}_{3}\right)$ : $\delta(\mathrm{ppm}) 197.1,151.0,149.3,136.5,135.9,130.6,129.0,128.5,26.4,16.1$. 


\subsubsection{0 methyl 4-(4-methylthiazol-5-yl)benzoate 3aj [3m]}

${ }^{1} \mathrm{H}$ NMR (400 MHz, $\left.\mathrm{CDCl}_{3}\right): \delta(\mathrm{ppm}) 8.71$ (s, Ar-H, 1H), 8.09 (m, Ar-H, 2H), 7.52 (m, Ar-H, 2H), $3.93\left(\mathrm{~s}, \mathrm{CH}_{3}, 3 \mathrm{H}\right), 2.55\left(\mathrm{~s}, \mathrm{CH}_{3}, 3 \mathrm{H}\right) .{ }^{13} \mathrm{C}$ NMR $\left(100 \mathrm{MHz}, \mathrm{CDCl}_{3}\right): \delta$ (ppm) 166.5, 151.0, 149.4, 136.6, 130.9, 129.9, 129.3, 129.0, 52.2, 16.3.

\subsubsection{4-methyl-5-(4-(trifluoromethyl)phenyl)thiazole 3ak [3m]}

${ }^{1} \mathrm{H}$ NMR $\left(400 \mathrm{MHz}, \mathrm{CDCl}_{3}\right): \delta(\mathrm{ppm}) 8.70(\mathrm{~s}, \mathrm{Ar}-\mathrm{H}, 1 \mathrm{H}), 7.66(\mathrm{~m}, \mathrm{Ar}-\mathrm{H}, 2 \mathrm{H}), 7.53$ (m, Ar-H, 2H), $2.52\left(\mathrm{~s}, \mathrm{CH}_{3}, 3 \mathrm{H}\right) .{ }^{13} \mathrm{C} \mathrm{NMR}\left(100 \mathrm{MHz}, \mathrm{CDCl}_{3}\right): \delta(\mathrm{ppm}) 151.0,149.4$, 135.6, 130.3, 129.8 (q, J=32.5 Hz), 129.4, 125.6 (q, J=3.6 Hz), 123.9 (q, J=270.4 Hz), 16.0.

\subsubsection{4-methyl-5-phenylthiazole 3al [3m]}

${ }^{1} \mathrm{H}$ NMR (400 MHz, $\mathrm{CDCl}_{3}$ ), $\delta$ (ppm): 8.69 (s, Ar-H, 1H), 7.45 (m, Ar-H, 4H), 7.38 (m, Ar-H, 1H), $2.54\left(\mathrm{~s}, \mathrm{CH}_{3}, 3 \mathrm{H}\right) .{ }^{13} \mathrm{C} \mathrm{NMR}\left(100 \mathrm{MHz}, \mathrm{CDCl}_{3}\right), \delta(\mathrm{ppm}): 150.2$, $148.4,131.9,131.9,129.3,128.7,127.9,16.0$.

\subsubsection{4-methyl-5-(p-tolyl)thiazole3am [2k]}

${ }^{1} \mathrm{H}$ NMR (400 MHz, $\mathrm{CDCl}_{3}$ ), $\delta$ (ppm): 8.70 (s, Ar-H, 1H), 7.39 (d, J=8.1 Hz, Ar-H, 2H), $7.30(\mathrm{~d}, \mathrm{~J}=8.1 \mathrm{~Hz}, \mathrm{Ar}-\mathrm{H}, 2 \mathrm{H}), 2.58\left(\mathrm{~s}, \mathrm{CH}_{3}, 3 \mathrm{H}\right), 2.45\left(\mathrm{~s}, \mathrm{CH}_{3}, 3 \mathrm{H}\right) .{ }^{13} \mathrm{C} \mathrm{NMR}$ $\left(100 \mathrm{MHz}, \mathrm{CDCl}_{3}\right), \delta$ (ppm): 150.0, 148.2, 137.9, 132.0, 129.4, 129.2, 129.0, 21.2, 16.0 .

\subsubsection{4-methyl-5-(m-tolyl)thiazole 3an [3m]}

${ }^{1} \mathrm{H}$ NMR (400 MHz, $\left.\mathrm{CDCl}_{3}\right): \delta$ (ppm) 8.53 (s, Ar-H, 1H), $7.20(\mathrm{~m}, \mathrm{Ar}-\mathrm{H}, 3 \mathrm{H}), 7.05$ (m, Ar-H, 1H), $2.42\left(\mathrm{~s}, \mathrm{CH}_{3}, 3 \mathrm{H}\right), 2.27\left(\mathrm{~s}, \mathrm{CH}_{3}, 3 \mathrm{H}\right) .{ }^{13} \mathrm{C} \mathrm{NMR}\left(100 \mathrm{MHz}, \mathrm{CDCl}_{3}\right): \delta$ (ppm) 150.0, 148.2, 138.2, 131.9, 131.6, 129.8, 128.5, 128.4, 126.2, 21.2, 15.9. 
4.3.15 4-methyl-5-(o-tolyl)thiazole 3ao [3m]

${ }^{1} \mathrm{H}$ NMR (400 MHz, $\mathrm{CDCl}_{3}$ ), $\delta$ (ppm): 8.74 (s, Ar-H, 1H), 7.32 (m, Ar-H, 2H), 7.25 (m, Ar-H, 2H), $2.30\left(\mathrm{~s}, \mathrm{CH}_{3}, 3 \mathrm{H}\right), 2.21\left(\mathrm{~s}, \mathrm{CH}_{3}, 3 \mathrm{H}\right) .{ }^{13} \mathrm{C}$ NMR (100 MHz, $\left.\mathrm{CDCl}_{3}\right), \delta$ (ppm): 151.0, 151.0, 149.7, 137.9, 131.3, 130.8, 130.3, 128.7, 125.7, 20.1, 15.4.

4.3.16 4-methyl-5-(naphthalen-1-yl)thiazole 3ap [3m]

${ }^{1} \mathrm{H}$ NMR (400 MHz, $\left.\mathrm{CDCl}_{3}\right): \delta(\mathrm{ppm}) 8.67$ (s, Ar-H, 1H), 7.75 (m, Ar-H, 2H), 7.55 (m, Ar-H, 1H), 7.36 (m, Ar-H, 4H), 2.14 (s, $\left.\mathrm{CH}_{3}, 3 \mathrm{H}\right) .{ }^{13} \mathrm{C}$ NMR (100 MHz, $\left.\mathrm{CDCl}_{3}\right)$ : $\delta(\mathrm{ppm}) 151.4,150.6,133.5,132.3,129.2,129.0,128.9,128.6,128.3,126.5,126.0$ 125.4, 125.0, 15.6.

4.3.17 5-(4-methoxyphenyl)-4-methylthiazole 3aq [2k]

${ }^{1} \mathrm{H}$ NMR (400 MHz, $\left.\mathrm{CDCl}_{3}\right): \delta(\mathrm{ppm}) 8.52(\mathrm{~s}, \mathrm{Ar}-\mathrm{H}, 1 \mathrm{H}), 7.26(\mathrm{~m}, \mathrm{Ar}-\mathrm{H}, 2 \mathrm{H}) 6.85(\mathrm{~m}$, $\mathrm{Ar}-\mathrm{H}, 2 \mathrm{H}), 3.72\left(\mathrm{~s}, \mathrm{CH}_{3}, 3 \mathrm{H}\right), 2.41\left(\mathrm{~s}, \mathrm{CH}_{3}, 3 \mathrm{H}\right) .{ }^{13} \mathrm{C} \mathrm{NMR}\left(100 \mathrm{MHz}, \mathrm{CDCl}_{3}\right): \delta$ (ppm) 159.2, 149.5, 147.7, 131.6, 130.3, 123.9, 114.0, 55.1, 15.8 .

4.3.18 5-(3-methoxyphenyl)-4-methylthiazole 3ar [2k]

${ }^{1} \mathrm{H}$ NMR (400 MHz, $\mathrm{CDCl}_{3}$ ): $\delta$ (ppm) 8.64 (m, Ar-H, 1H), 7.32 (m, Ar-H, 1H), 7.00 (m, Ar-H, 2H), 6.89 (m, Ar-H, 1H), $3.80\left(\mathrm{~s}, \mathrm{CH}_{3}, 3 \mathrm{H}\right), 2.52\left(\mathrm{~s}, \mathrm{CH}_{3}, 3 \mathrm{H}\right) .{ }^{13} \mathrm{C} \mathrm{NMR}$ (100 MHz, $\left.\mathrm{CDCl}_{3}\right): \delta(\mathrm{ppm}) 159.5,150.1,148.4,133.0,131.6,129.6,121.6,114.9$, 113.1, 55.1, 16.0.

4.3.19 4-(2, 4-dimethylthiazol-5-yl)benzonitrile 3ba [22]

${ }^{1} \mathrm{H}$ NMR (400 MHz, $\mathrm{CDCl}_{3}$ ), $\delta$ (ppm): 7.68 (m, Ar-H, 2H), 7.50 (m, Ar-H, 2H), 2.68 (s, $\left.\mathrm{CH}_{3}, 3 \mathrm{H}\right), 2.46\left(\mathrm{~s}, \mathrm{CH}_{3}, 3 \mathrm{H}\right) .{ }^{13} \mathrm{C} \mathrm{NMR}\left(100 \mathrm{MHz}, \mathrm{CDCl}_{3}\right), \delta$ (ppm): 164.7, 148.7, $137.1,132.3,129.4,129.3,118.5,110.9,19.1,16.3$. 


\subsubsection{5-(4-chlorophenyl)-2, 4-dimethylthiazole $3 \boldsymbol{b b}$}

${ }^{1} \mathrm{H}$ NMR (400 MHz, $\mathrm{CDCl}_{3}$ ), $\delta$ (ppm): 7.35 (m, Ar-H, 4H), 2.65 (s, $\left.\mathrm{CH}_{3}, 3 \mathrm{H}\right), 2.41$ (s, $\left.\mathrm{CH}_{3}, 3 \mathrm{H}\right) .{ }^{13} \mathrm{C} \mathrm{NMR}\left(100 \mathrm{MHz}, \mathrm{CDCl}_{3}\right), \delta(\mathrm{ppm}): 163.5,147.3,133.5,130.7,130.2$, 130.0, 128.7, 19.0, 15.9. ESI-MS. $m / z: 224\left[\mathrm{M}^{+}\right]$

\subsubsection{4-(2, 4-dimethylthiazol-5-yl)benzaldehyde 3bc}

${ }^{1} \mathrm{H}$ NMR (400 MHz, $\mathrm{CDCl}_{3}$ ), $\delta$ (ppm): 10.01 (s, CHO, 1H), 7.91 (m, Ar-H, 2H), 7.57 (m, Ar-H, 2H), 2.69 (s, $\left.\mathrm{CH}_{3}, 3 \mathrm{H}\right), 2.49\left(\mathrm{~s}, \mathrm{CH}_{3}, 3 \mathrm{H}\right) .{ }^{13} \mathrm{C} \mathrm{NMR}\left(100 \mathrm{MHz}, \mathrm{CDCl}_{3}\right), \delta$ (ppm): 191.4, 164.6, 148.5, 138.6, 135.0, 130.1, 130.0, 129.3, 19.1, 16.4. ESI-MS. $m / z: 218\left[\mathrm{M}^{+}\right]$

\subsubsection{2 methyl 4-(2, 4-dimethylthiazol-5-yl)benzoate $3 \boldsymbol{b j}$}

${ }^{1} \mathrm{H}$ NMR (400 MHz, CDCl 3 ), $\delta$ (ppm): 8.05 (m, Ar-H, 2H), 7.46 (m, Ar-H, 2H), 3.91 (m, $\left.\mathrm{CH}_{3}, 3 \mathrm{H}\right), 2.67\left(\mathrm{~s}, \mathrm{CH}_{3}, 3 \mathrm{H}\right), 2.47\left(\mathrm{~m}, \mathrm{CH}_{3}, 3 \mathrm{H}\right) .{ }^{13} \mathrm{C} \mathrm{NMR}\left(100 \mathrm{MHz}, \mathrm{CDCl}_{3}\right), \delta$ (ppm): 166.5, 164.1, 148.1, 137.0, 130.3, 129.8, 128.9, 128.7, 52.1, 19.1, 16.3. ESI-MS. $m / z: 248\left[\mathrm{M}^{+}\right]$

\subsubsection{2, 4-dimethyl-5-phenylthiazole $3 \boldsymbol{b l}[2 g]$}

${ }^{1} \mathrm{H}$ NMR (400 MHz, CDCl $), \delta(\mathrm{ppm}): 7.38$ (m, Ar-H, 4H), 7.31 (m, Ar-H, 1H), 2.65 (s, $\left.\mathrm{CH}_{3}, 3 \mathrm{H}\right), 2.44$ (s, $\left.\mathrm{CH}_{3}, 3 \mathrm{H}\right) .{ }^{13} \mathrm{C} \mathrm{NMR}\left(100 \mathrm{MHz}, \mathrm{CDCl}_{3}\right), \delta$ (ppm): 163.1, 146.9, $132.2,131.2,129.0,128.5,127.4,18.9,15.9$.

\subsubsection{2, 4-dimethyl-5-(p-tolyl)thiazole 3bm [23]}

${ }^{1} \mathrm{H}$ NMR (400 MHz, CDCl $), \delta$ (ppm): 7.29 (m, Ar-H, 2H), 7.20 (m, Ar-H, 2H), 2.65 (s, $\left.\mathrm{CH}_{3}, 3 \mathrm{H}\right), 2.44\left(\mathrm{~s}, \mathrm{CH}_{3}, 3 \mathrm{H}\right), 2.36\left(\mathrm{~s}, \mathrm{CH}_{3}, 3 \mathrm{H}\right) .{ }^{13} \mathrm{C} \mathrm{NMR}\left(100 \mathrm{MHz}, \mathrm{CDCl}_{3}\right), \delta$ (ppm): 162.7, 146.5, 137.2, 131.2, 129.2, 129.2, 128.8, 21.0, 18.9, 15.8. 


\section{Acknowledgements}

The authors gratefully thank the Foundation for Distinguished Young Talents in Higher Education of Guangdong and National Science Foundation of China (No. Yq2013101, No. 51203026, No. 2012-LYM0080) for the financial support.

\section{References}

[1] Recent reviews on direct $\mathrm{C}-\mathrm{H}$ arylation, see: (a) L.C. Campeau, R. Stuart, K. Fagnou, Aldrichimica Acta. 40 (2007) 35-41; (b) I.V. Seregin, V. Gevorgyan, Chem. Soc. Rev. 36 (2007) 1173-1193; (c) D. Alberico, M.E. Scott, M. Lautens, Chem. Rev. 107 (2007) 174-238; (d) L. Ackermann, R. Vicente, A.R. Kapdi, Angew. Chem. Int. Ed. 48 (2009) 9792-9826; (e) X. Chen, K.M. Engle, D.-H. Wang, J.-Q. Yu, Angew. Chem. Int. Ed. 48 (2009) 5094-5115; (f) F. Bellina, R. Rossi, Tetrahedron 65 (2009) 10269-10310; (g) J. Roger, A.L. Gottumukkala, H. Doucet, Chem. Cat. Chem. 2 (2010) 20-40; (h) J. Wencel-Delord, T. Dröge, F. Liu, F. Glorius, Chem. Soc. Rev. 40 (2011) 4740-4761; (i) L. Ackermann, Chem. Rev. 111 (2011) 1315-1345; (j) C.-L. Sun, B.-J. Li, Z.-J. Shi, Chem. Rev. 111 (2011) 1293-1314; (k) D. Zhao, J. You, C. Hu, Chem. Eur. J. 17 (2011) 5466-5492; (1) J. Yamaguchi, A.D. Yamaguchi, K. Itami, Angew. Chem., Int. Ed. 51 (2012) 8960-9009; (m) T. Brückl, R.D. Baxter, Y. Ishihara, P.S. Baran, Acc. Chem. Res. 45 (2012) 826-839; (n) R. Rossi, F. Bellina, M. Lessi, C. Manzinia, Adv. Synth. Catal. 356 (2014) 17-117; (o) M. He, J.-F. Soulé, H. Doucet, Chem.Cat.Chem. 6 (2014) 1824-1859; (p) I. Hussain, T. Singh, Adv. Synth. Catal. 356 (2014) 
1661-1696; (q) K. Yuan, J.-F. Soulé, H. Doucet, ACS Catal. 5 (2015) 978-991; (r)

S.E. Kazzouli, J. Koubachi, N.E. Brahmi, G. Guillaumet. RSC Adv. 5 (2015) $15292-15327$.

[2] For selected examples on the arylation of thiazoles promoted by phosphine-based palladium complexes, see: (a) Y. Aoyagi, A. Inoue, I. Koizumi, R. Hashimoto, K. Tokunaga, K. Gohma, J. Komatsu, K. Sekine, A. Miyafuji, J. Kunoh, R. Honma, Y. Akita, A. Ohta, Heterocycles 33 (1992) 257-272; (b) S. Pivsa-Art, T. Satoh, Y. Kawamura, M. Miura, M. Nomura, Bull. Chem. Soc. Jpn. 71 (1998) 476-473; (c) A. Mori, A. Sekiguchi, K. Masui, T. Shimada, M. Horie, K. Osakada, M. Kawamoto, T. Ikeda, J. Am. Chem. Soc. 125 (2003) 1700-1701; (d) A. Yokooji, T. Okazawa, T. Satoh, M. Miura, M. Nomura, Tetrahedron 59 (2003) 5685-5689; (e) K. Masui, A. Mori, K. Okano, K. Takamura, M. Kinoshita, T. Ikeda, Org. Lett. 6 (2004) 2011-2014; (f) K. Kobayashi, M.S. M. Ahmed , A. Mori, Tetrahedron 62 (2006) 9548-9553; (g) G.L. Turner, J.A. Morris , M.F. Greaney, Angew. Chem. Int. Ed. 46 (2007) 7996-8000; (h) H.A. Chiong, O. Daugulis, Org. Lett. 9 (2007) 1449-1451; (i) T. Martin, C. Verrier, C. Hoarau , F. Marsais, Org. Lett. 10 (2008) 2909-2912; (j) F. Derridj, A. L. Gottumukkala, S. Djebbar, H. Doucet, Eur. J. Inorg. Chem. 16 (2008) 2550-2559; (k) B. Liégault, D. Lapointe, L. Caron, A. Vlassova, K. Fagnou, J. Org. Chem. 74 (2009) 1826-1834; (1) N. Primas, A. Bouillon, J.-C. Lancelot, H. EI-Kashef, S. Rault, Tetrahedron 65 (2009) 5739-5746; (m) S. Tamba, Y. Okubo, S. Tanaka, D. Monguchi, A. Mori, J. Org. Chem. 75 (2010) 6998-7001; (n) B. Liégault, I. Petrov, S.I. Gorelsky, K. Fagnou, 
J. Org. Chem. 75 (2010) 1047-1060; (o) D. Roy, S. Mom, M. Beaupérin, H. Doucet, J.-C. Hierso, Angew. Chem., Int. Ed. 49 (2010) 6650-6654; (p) J. J. Dong, J. Roger, C. Verrier, T. Martin, R. Le Goff, C. Hoarau, H. Doucet, Green Chem. 12 (2010) 2053-2063; (q) D. Lapointe, T. Markiewicz, C.J. Whipp, A. Toderian, K. Fagnou, J. Org. Chem. 76 (2011) 749-759; (r) M. Baghbanzadeh, C. Pilger, C.O. Kappe, J. Org. Chem. 76 (2011) 8138-8142; (s) K, Beydoun, J. Roger, J. Boixel, H.L. Bozec, V. Guerchais, H. Doucet, Chem. Commun. 48 (2012) 11951-11953; (t) F. Belkessam, F. Derridj, L. Zhao, A. Elias, M. Aidene, S. Djebbar, H. Doucet, Tetrahedron Lett. 54 (2013) 4888-4891; (u) F. Belkessam, A. Mohand, J.-F. Soulé, A. Elias, H. Doucet, Beilstein J. Org. Chem. 10 (2014) 2912-2919; (v) I. Smari, L. Zhao, K. Yuan, H.B. Ammar, H. Doucet, Catal. Sci. Technol. 4 (2014) 3723-3732; (w) P. Chaveź, C. Ngov, P. de Frémon, P. Lévêque, N. Leclerc, J. Org. Chem.79 (2014) 10179-10188; (x) A. Jakab, Z. Dalicsek, T. Soós, Eur. J. Org. Chem. (2015) 56-59.

[3] For selected examples on the arylation of thiazoles promoted by phosphine-free and ligand-free palladium complexes, see: (a) Y. Kondo, T. Komine, T. Sakamoto, Org. Lett. 2 (2000) 3111-3113; (b) M. Parisien, D. Valette, K. Fagnou, J. Org. Chem. 70 (2005) 7578-7584; (c) F. Bellina, S. Cauteruccio, R. Rossi, Eur. J. Org. Chem. (2006) 1379-1382; (d) J. Roger, F. Požgan, H. Doucet, J. Org. Chem. 74 (2009) 1179-1186; (e) I. Ozdemir, Y. Gök, Ö. Özeroğlu, M. Kaloğlu, H. Doucet, C. Bruneau, Eur. J. Inorg. Chem. (2010) 1798-1805; (f) F. Shibahara, E. Yamaguchi, T. Murai, J. Org. Chem. 76 (2011) 2680-2693; (g) S. 
Yanagisawa, K. Itami, Tetrahedron 67 (2011) 4425-4433; (h) S. Kirchberg, S. Tani, K. Ueda, J. Yamaguchi, A. Studer, K. Itami, Angew. Chem. Int. Ed. 50 (2011) 2387-2391. (i) F. Shibahara, T. Yamauchi, E. Yamaguchi, T. Murai, J. Org. Chem. 77 (2012) 8815-8820; (j) S. Bensaid, H. Doucet, Chem.Sus.Chem. 5 (2012) 1559-1567; (k) T.N. Uehara, J. Yamaguchi, K. Itami, Asian J. Org. Chem. 2 (2013) 938-942; (1) F. Bellina, M. Lessi, C. Manzini, Eur. J. Org. Chem. (2013) 5621-5630; (m) X.-W. Liu, J.-L. Shi, J.-X. Yan, J.-B. Wei, K. Peng, L. Dai, C.-G. Li, B.-Q. Wang, Z.-J. Shi, Org. Lett. 15 (2013) 5774-5777; (n) L. Chen, C. Bruneau, P.H. Dixneuf, H. Doucet, Chem.Cat.Chem. 5 (2013) 1956-1963; (o) S.K. Kim, J.-H. Kim, Y.C. Park, J.W. Kim, E.K. Yum, Tetrahedron 69 (2013) 10990-10995; (p) S. Akkoç, Y. Gök, Appl. Organomet. Chem. 28 (2014) 854-860; (q) S. Akkoç, Y. Gök, M. Akkurt, M. N. Tahir, Inorg. Chim. Acta. 413 (2014) 221-230; (r) H, Sekizawa, K. Amaike, Y. Itoh, T. Suzuki, K. Itami, J. Yamaguchi, ACS Med. Chem. Lett. 5 (2014) 582-586; (s) S. Tani, T.N. Uehara, J. Yamaguchi, K. Itami, Chem. Sci. 5 (2014) 123-135; (t) S. Akkoç, Y. Gök, Inorg. Chim. Acta. 429 (2015) 34-38.

[4] For selected examples on the arylation of thiazoles promoted by other metal complexes, see: (a) B. Sezen, D. Sames, 5 (2003) 3607-3610; (b) H.-Q. Do, R.M.K. Khan, O. Daugulis, J. Am. Chem. Soc. 130 (2008) 15185-15192; (n) J. Canivet, J. Yamaguchi, I. Ban, K. Itami, Org. Lett. 11 (2009) 1733-1736; (c) H. Hachiya, K. Hirano, T. Satoh, M. Miura, Angew. Chem. Int. Ed. 49 (2010) 2202-2205; (d) T. Yamamoto, K. Muto, M. Komiyama, J. Canivet, J. Yamaguchi, 
K. Itami, Chem. Eur. J. 17 (2011) 10113-10122; (e) K. Muto, J. Yamaguchi, K. Itami, J. Am. Chem. Soc. 134 (2012) 169-172; (f) K. Amaike, K. Muto, J. Yamaguchi , K. Itami, J. Am. Chem. Soc. 134 (2012) 13573-13576.

[5] (a) S. Miwatashi, Y. Arikawa, E. Kotani, M. Miyamoto, K. Naruo, H. Kimura, T. Tanaka, S. Asahi,S. Ohkawa, J. Med. Chem. 48 (2005) 5966-5979. (b) J. Lee, S.J. Kim, H. Choi, Y.H. Kim, I.T. Lim, H. Yang, C.S. Lee, H.R. Kang, S.K. Ahn, S.K. Moon, D.H. Kim, S. Lee, N.S. Choi, K.J. Lee, J. Med. Chem. 53 (2010) 6337-6354; (c) D. Zwilling, S.-Y. Huang, K.V. Sathyasaikumar, F.M. Notarangelo, P. Guidetti, H.-Q. Wu, J. Lee, J. Truong, Y. Andrews-Zwilling, E.W. Hsieh, J.Y. Louie, T. Wu, K. Scearce-Levie, C. Patrick, A. Adame, F. Giorgini, S. Moussaoui, G. Laue, A. Rassoulpour, G. Flik, Y. Huang, J.M. Muchowski, E. Masliah, R. Schwarcz, P. J. Muchowski, Cell 145 (2011) 863-874; (d) L.G. Mercier, M. Leclerc, Acc. Chem. Res. 46 (2013) 1597-1605.

[6] Understanding Organometallic Reaction Mechanisms and Catalysis: Computational and Experimental Tool, ed. V.P. Ananikov, Wiley-VCH, Weinheim, 1st edn, 2015.

[7] (a) K.M. Engle, T.-S. Mei, M. Wasa, J.-Q. Yu, Acc. Chem. Res. 45 (2012) 788-802; (b) K. M. Engle, J.-Q. Yu, J. Org. Chem. 78 (2013) 8927-8955. (c) F. Shibahara, T. Murai, Asian J. Org. Chem. 2 (2013) 624-636; (d) S.M. Wong, O.Y. Yuen, P.Y. Choy, F.Y. Kwong, Coord. Chem. Rev. 293-294 (2015) 158-186..

[8] (a) A.F. Littke, G.C. Fu, Angew. Chem. Int. Ed. 41(2002) 4176-4211; (b)R. Martin, S.L. Buchwald, Acc. Chem. Res. 41 (2008) 1461-1473. 
[9] (a) B. Tao, D.W. Boykin, Tetrahedron Lett. 44 (2003) 7993-7996; (b) B. Tao, D.W. Boykin, J. Org. Chem. 69 (2004) 4330-4335; (c) J.-H. Li, W.-J. Liu, Org. Lett. 6 (2004) 2809-2811; (d) J.M. Chalker, C.S.C. Wood, B.G. Davis, J. Am. Chem. Soc. 131 (2009) 16346-16347; (e) P. Das, C. Sarmah, A. Tairai, U. Bora, Appl. Orgaomet. Chem. 25 (2011) 283-288; (f) I. Cobo, M.I. Matheu, S. Castillón, O. Boutureira, B. G. Davis, Org. Lett. 14 (2012) 1728-1731; (g) C.D. Spicer, T. Triemer, B.G. Davis, J. Am. Chem. Soc. 134 (2012) 800-803; (h) C. Liu, Y. Zhang, N. Liu, J. Qiu, Green Chem. 14 (2012) 2999-3003; (i) T. Abe, T. Mino, K. Watanabe, F. Yagishita, M. Sakamoto, Eur. J. Org. Chem. (2014) 3909-3916. [10](a) T.E. Storr, C.G. Baumann, R.J. Thatcher, S.D. Ornellas, A.C. Whitwood, I.J.S. Fairlamb, J. Org. Chem. 74 (2009) 5810-5821; (b) S.E. Bajwa, T.E. Storr, L. E. Hatcher, T. J. Williams, C.G. Baumann, A.C. Whitwood, D.R. Allan, S.J. Teat, P.R. Raithby, I.J.S. Fairlamb, Chem. Sci. 3 (2012) 1656-1661; (c) C.G. Baumann, S.D. Ornellas, J.P. Reeds, T.E. Storr, T.J. Williams, I.J.S. Fairlamb, Tetrahedron 70 (2014) 6174-6187.

[11]T. Iwasawa, M. Tokunaga, Y. Obora, Y. Tsuji, J. Am. Chem. Soc. 126 (2004), $6554-6555$.

[12](a) G. Berthon-Gelloz, M.A. Siegler, A.L. Spek, B. Tinant, J.N.H. Reek, I.E. Markó, Dalton Trans. 39 (2010) 1444-1446; (b) S. Meiries, K. Speck, D.B. Cordes, A.M.Z. Slawin, S.P. Nolan, Organometallics 32 (2013) 330-339.

[13]L. Chahen, B. Therrien, G. Süss-Fink, Eur. J. Inorg. Chem. (2007) 5045-5051.

[14](a) Y. Li, J. Wang, M. Huang, Z. Wang, Y. Wu, Y. Wu, J. Org. Chem. 79 (2014) 
2890-2897; (b) D.S. Lee, P.Y. Choy, C. M. So, J. Wang, C.P. Lau, F.Y. Kwong, RSC Adv. 2 (2012) 9179-9182.

[15](a) D. Lapointe, K. Fagnou, Chem. Lett. 39 (2010) 1118-1126; (b) S. I. Gorelsky, Coord. Chem. Rev. 257 (2013) 153-164; (c) A review of carboxylate-assited metal-catalyzed C-H bond functinalizations, seen in reference $1 \mathrm{~h}$.

[16](a) Applied Homogeneous Catalysis with Organometallic Compounds, ed. B. Cornils, and W. A. Herrmann, Wiley-VCH, Weinheim, 2nd edn, 2002; (b) G.C. Fortman, S.P. Nolan, Chem. Soc. Rev. 40 (2011) 5151-5169; (c) Homogeneous Catalysis for Unreactive Bond Activation, ed. Z.-J. Shi, Wiley-VCH, Weinheim, 1st edn, 2014.

[17](a) H.Y. Fu, L. Chen, H. Doucet, J. Org. Chem. 77 (2012) 4473-4478; (b) L. Basolo, E.M. Beccalli, E. Borsini, G. Broggini, Tetrahedron 65 (2009) 3486-3491; (c) D. Zhao, W. Wang, S. Lian, F. Yang, J. Lan, J. You, Chem.-Eur. J. 15 (2009) 1337-1340.

[18](a) A.H.M. de Vries, J.M.C.A. Mulders, J.H.M. Mommers, H.J.W. Henderickx, J.G. de Vries, Org. Lett. 5 (2003) 3285-3288; (b) M.T. Reetz, J.G.J. G. de Vries, Chem. Commun. (2004) 1559-1563; (c) D. Saha, L. Adak, B.C. Ranu, Tetradedron Lett. 51 (2010) 5624-5627.

[19](a) J.A. Widegren, R.G. Finke, J. Mol. Catal. A: Chem. 198 (2003) 317-341; (b) R.H. Crabtree, Chem. Rev. 112 (2012) 1536-1554.

[20](a) M. Lafrance, K. Fagnou, J. Am. Chem. Soc. 128(2006) 16496-16497; (b) H.-Y. Sun, S. I. Gorelsky, D. R. Stuart, L.-C. Campeau, K. Fagnou, J. Org. Chem. 75 
(2010) 8180-8189.

[21]A. Cohen, M.D. Crozet, P. Rathelot, P. Vanelle, Green Chem. 11 (2009) $1736-1742$.

[22]K. Cooper, M.J. Fray, M. J. Parry, K. Richardson, J. Steele, J. Med. Chem. 35 (1992) 3115-3129.

[23]L. J. Gooßen, P.P. Lange, N. Rodriguez, C. Linder, Chem.-Eur. J. 16 (2010) 3906-3909.

\section{Figures}

Figure 1 Molecular structure of C2 depicted with $30 \%$ thermal ellipsoids. Hydrogen atoms except on the amines and one half of the non-coordinated methanol molecule have been omitted for clarity. Selected bond distances $(\AA)$ : $\mathrm{Pd}(1)-\mathrm{N}(1)$ 2.056(3), $\mathrm{Pd}(1)-\mathrm{N}(2)$ 2.056(3), $\mathrm{Pd}(1)-\mathrm{Cl}(1)$ 2.2914(10), $\mathrm{Pd}(1)-\mathrm{Cl}(2)$ 2.2938(9).

Figure 2 Molecular structure of $\mathbf{C 3}$ depicted with $30 \%$ thermal ellipsoids. Hydrogen atoms except on the amines and four non-coordinated dichloromethane molecules have been omitted for clarity. Selected bond distances $\quad(\AA): \quad \operatorname{Pd}(1)-\mathrm{N}(1) \quad 2.073(4), \quad \operatorname{Pd}(1)-\mathrm{N}(2) \quad 2.072(4), \quad \operatorname{Pd}(1)-\mathrm{Cl}(1)$ 2.2752(13), $\mathrm{Pd}(1)-\mathrm{Cl}(2) 2.2764(13)$.

Figure 3 Molecular structure of C4 depicted with $30 \%$ thermal ellipsoids. Hydrogen atoms except on the amines have been omitted for clarity. Selected 
bond distances (丹): $\quad \operatorname{Pd}(1)-\mathrm{N}(1) \quad 2.0644(15), \quad \operatorname{Pd}(1)-\mathrm{N}(1 \mathrm{~A}) \quad 2.0644(15)$, $\operatorname{Pd}(1)-\mathrm{Cl}(1) 2.3023(5), \operatorname{Pd}(1)-\mathrm{Cl}(1 \mathrm{~A}) 2.3023(5)$.

Figure 4 Molecular structure of C5 depicted with 30\% thermal ellipsoids. Hydrogen atoms except on the amines have been omitted for clarity. Selected bond distances (丹): $\quad \operatorname{Pd}(1)-\mathrm{N}(1) \quad 2.0615(19), \quad \operatorname{Pd}(1)-\mathrm{N}(1 \mathrm{~A}) \quad 2.0615(19)$, $\operatorname{Pd}(1)-\mathrm{Cl}(1) 2.2877(6), \operatorname{Pd}(1)-\mathrm{Cl}(1 \mathrm{~A}) 2.2877(6)$.

\section{Tables}

Table 1 Screening of palladium complexes on direct arylation reaction of 4-methylthiazole with 4-bromobenzonitrile ${ }^{\mathrm{a}}$

Table 2 Screening of reaction conditions for the direct arylation reaction of 4-methylthiazole with 4-bromobenzonitrile

Table 3 The palladium-catalyzed direct arylation of substituted thiazoles with aryl bromides $^{\mathrm{a}}$

Table 4 The direct arylation of substituted thiazoles with aryl bromides at low palladium loading ${ }^{\mathrm{a}}$

\section{Schemes}

Scheme 1 Structure of the aniline palladium complexes

Scheme 2 Proposed catalytic cycle for direct arylation of thiazoles with aryl bromides 


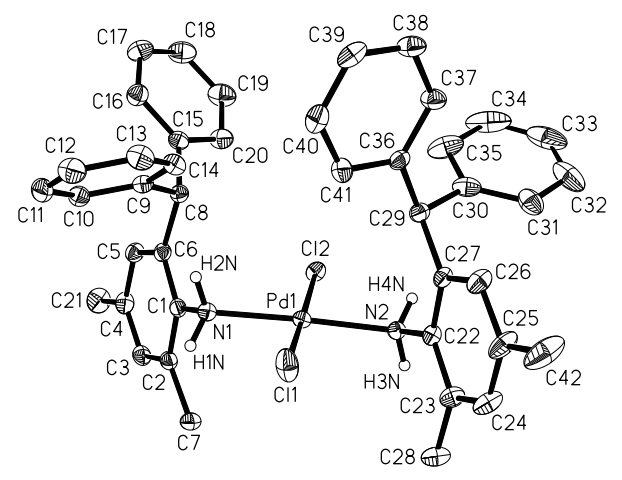

Figure 1 Molecular structure of C2 depicted with 30\% thermal ellipsoids. Hydrogen atoms except on the amines and one half of the non-coordinated methanol molecule have been omitted for clarity. Selected bond distances $(\AA)$ : $\mathrm{Pd}(1)-\mathrm{N}(1)$ 2.056(3), $\mathrm{Pd}(1)-\mathrm{N}(2)$ 2.056(3), $\mathrm{Pd}(1)-\mathrm{Cl}(1) 2.2914(10), \mathrm{Pd}(1)-\mathrm{Cl}(2)$ $2.2938(9)$ 


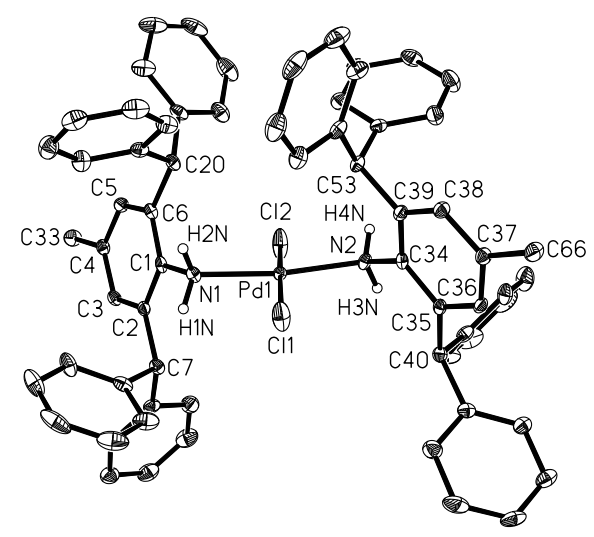

Figure 2 Molecular structure of C3 depicted with $30 \%$ thermal ellipsoids. Hydrogen atoms except on the amines and four non-coordinated dichloromethane molecules have been omitted for clarity. Selected bond distances $(\AA): \quad \operatorname{Pd}(1)-\mathrm{N}(1) \quad 2.073(4), \quad \operatorname{Pd}(1)-\mathrm{N}(2) \quad 2.072(4), \quad \operatorname{Pd}(1)-\mathrm{Cl}(1)$ 2.2752(13), $\mathrm{Pd}(1)-\mathrm{Cl}(2) 2.2764(13)$. 


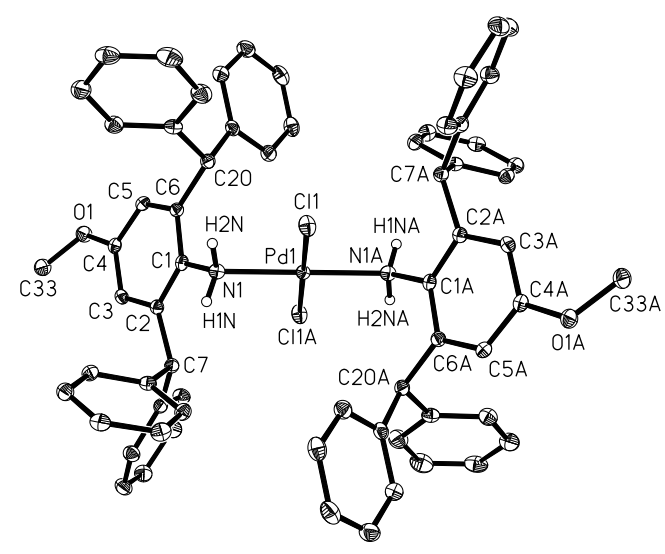

Figure 3 Molecular structure of C4 depicted with $30 \%$ thermal ellipsoids. Hydrogen atoms except on the amines have been omitted for clarity. Selected


$\operatorname{Pd}(1)-\mathrm{Cl}(1) 2.3023(5), \operatorname{Pd}(1)-\mathrm{Cl}(1 \mathrm{~A}) 2.3023(5)$. 


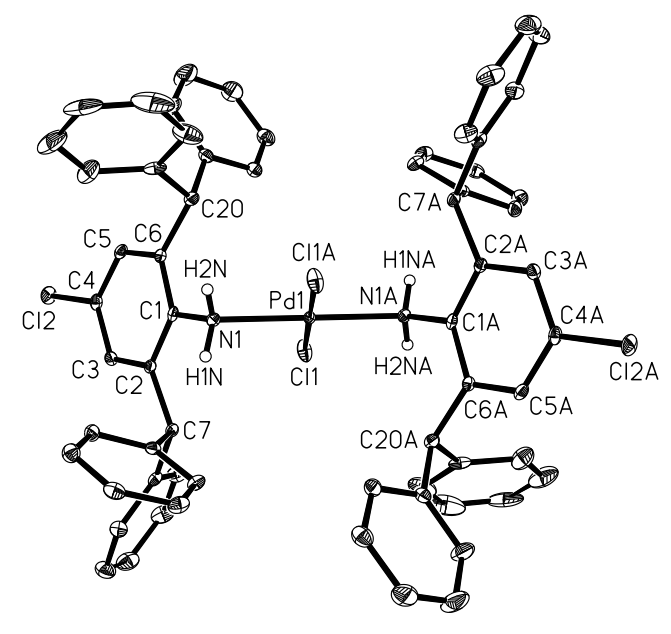

Figure 4 Molecular structure of C5 depicted with 30\% thermal ellipsoids.

Hydrogen atoms except on the amines have been omitted for clarity. Selected bond distances $(\AA): \quad \operatorname{Pd}(1)-\mathrm{N}(1) \quad 2.0615(19), \quad \operatorname{Pd}(1)-\mathrm{N}(1 \mathrm{~A}) \quad 2.0615(19)$, $\operatorname{Pd}(1)-\mathrm{Cl}(1) 2.2877(6), \operatorname{Pd}(1)-\mathrm{Cl}(1 \mathrm{~A}) 2.2877(6)$. 
Table 1 Screening of palladium complexes on direct arylation reaction of 4-methylthiazole with 4-bromobenzonitrile ${ }^{\mathrm{a}}$<smiles>Cc1ncsc1[18OH]</smiles>

1a



2a

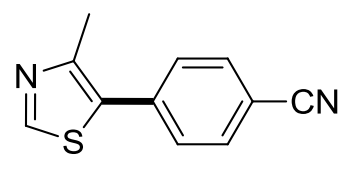

3aa

\begin{tabular}{cccc}
\hline Entry & Catalyst & $\mathrm{T}\left({ }^{\circ} \mathrm{C}\right)$ & Cov. $(\%)^{\mathrm{b}}$ \\
1 & $\mathbf{2 C y}_{\mathbf{2}} \mathbf{N H} / \mathbf{P d C l}_{\mathbf{2}}$ & 80 & 86 \\
2 & $\mathbf{C 1}$ & 80 & 85 \\
3 & $\mathbf{C 2}$ & 80 & $96(92)^{\mathrm{c}}$ \\
4 & $\mathbf{C 3}$ & 80 & 90 \\
5 & $\mathbf{C 4}$ & 80 & 91 \\
6 & $\mathbf{C 5}$ & 80 & 89 \\
\hline
\end{tabular}

${ }^{a}$ Reaction conditions: 4-methylthiazole $(1 \mathrm{mmol})$, 4-bromobenzonitrile (1 mmol), palladium source $(0.5 \mathrm{~mol} \%)$, PivOH $(0.3 \mathrm{mmol})$, base $(1.5 \mathrm{mmol})$, DMAc (3 mL) under aerobic environment for $24 \mathrm{~h} .{ }^{\mathrm{b}} \mathrm{GC}$ yield using the (trifluoromethyl)benzene as internal standard. 'isolated yields in parentheses. 
Table 2 Screening of reaction conditions for the direct arylation reaction of 4-methylthiazole with 4-bromobenzonitrile ${ }^{\mathrm{a}}$

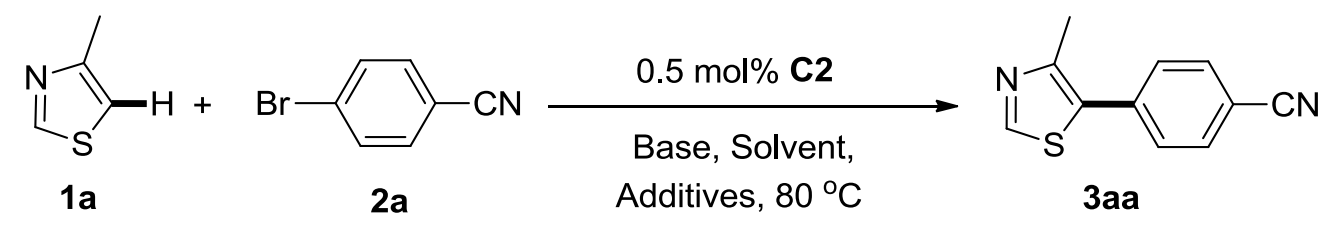

\begin{tabular}{|c|c|c|c|c|}
\hline Entry & Base & Solvent & Additive & Cov. $(\%)^{\mathrm{b}}$ \\
\hline 1 & $\mathrm{~K}_{2} \mathrm{CO}_{3}$ & DMAc & $\mathrm{PivOH}$ & 96 \\
\hline 2 & $\mathrm{Na}_{2} \mathrm{CO}_{3}$ & DMAc & $\mathrm{PivOH}$ & 47 \\
\hline 3 & $\mathrm{NaHCO}_{3}$ & DMAc & $\mathrm{PivOH}$ & 47 \\
\hline 4 & $\mathrm{C}_{6} \mathrm{H}_{5} \mathrm{CO}_{2} \mathrm{Na}$ & DMAc & $\mathrm{PivOH}$ & 29 \\
\hline 5 & $\mathrm{~K}_{3} \mathrm{PO}_{4}$ & DMAc & $\mathrm{PivOH}$ & 71 \\
\hline 6 & KOAc & DMAc & $\mathrm{PivOH}$ & 90 \\
\hline 7 & $\mathrm{NaOH}$ & DMAc & $\mathrm{PivOH}$ & 6 \\
\hline 8 & $\mathrm{NaOAc}$ & DMAc & $\mathrm{PivOH}$ & 30 \\
\hline 9 & $\mathrm{Cs}_{2} \mathrm{CO}_{3}$ & DMAc & $\mathrm{PivOH}$ & 5 \\
\hline 10 & $\mathrm{KOH}$ & DMAc & $\mathrm{PivOH}$ & NR \\
\hline 11 & $\mathrm{KO}^{\mathrm{t}} \mathrm{Bu}$ & DMAc & $\mathrm{PivOH}$ & NR \\
\hline 12 & $\mathrm{NaO}^{t} \mathrm{Bu}$ & DMAc & $\mathrm{PivOH}$ & NR \\
\hline 13 & $\mathrm{~K}_{2} \mathrm{CO}_{3}$ & DMF & $\mathrm{PivOH}$ & 84 \\
\hline 14 & $\mathrm{~K}_{2} \mathrm{CO}_{3}$ & Dioxane & $\mathrm{PivOH}$ & 70 \\
\hline 15 & $\mathrm{~K}_{2} \mathrm{CO}_{3}$ & Toluene & $\mathrm{PivOH}$ & 50 \\
\hline 16 & $\mathrm{~K}_{2} \mathrm{CO}_{3}$ & Xylene & $\mathrm{PivOH}$ & 39 \\
\hline 17 & $\mathrm{~K}_{2} \mathrm{CO}_{3}$ & NMP & $\mathrm{PivOH}$ & 62 \\
\hline 18 & $\mathrm{~K}_{2} \mathrm{CO}_{3}$ & DMSO & $\mathrm{PivOH}$ & NR \\
\hline 19 & $\mathrm{~K}_{2} \mathrm{CO}_{3}$ & $\mathrm{EtOH}$ & $\mathrm{PivOH}$ & NR \\
\hline 20 & $\mathrm{~K}_{2} \mathrm{CO}_{3}$ & DMAc & - & 9 \\
\hline 21 & $\mathrm{~K}_{2} \mathrm{CO}_{3}$ & DMAc & HOAc & 67 \\
\hline 22 & $\mathrm{~K}_{2} \mathrm{CO}_{3}$ & DMAc & $\mathrm{C}_{2} \mathrm{H}_{5} \mathrm{COOH}$ & 66 \\
\hline 23 & $\mathrm{~K}_{2} \mathrm{CO}_{3}$ & DMAc & $\mathrm{CF}_{3} \mathrm{COOH}$ & 14 \\
\hline 24 & $\mathrm{~K}_{2} \mathrm{CO}_{3}$ & DMAc & Lactic acid & 15 \\
\hline 25 & $\mathrm{~K}_{2} \mathrm{CO}_{3}$ & DMAc & $\mathrm{CH}_{2}(\mathrm{COOH})_{2}$ & 19 \\
\hline 26 & $\mathrm{~K}_{2} \mathrm{CO}_{3}$ & DMAc & o-Phthalic Acid & 11 \\
\hline 27 & $\mathrm{~K}_{2} \mathrm{CO}_{3}$ & DMAc & Benzoic acid & 32 \\
\hline
\end{tabular}

${ }^{\mathrm{a}}$ Conditions: 1a $(1.0 \mathrm{mmol})$, 4-bromobenzonitrile $(1.0 \mathrm{mmol}), \mathbf{C 2}(0.5 \mathrm{~mol} \%)$, Additives $(0.3 \mathrm{mmol})$, base $(1.5 \mathrm{mmol})$, solvent $(3 \mathrm{~mL}), 80{ }^{\circ} \mathrm{C}, 24 \mathrm{~h} .{ }^{\mathrm{b}} \mathrm{GC}$ yield using the (trifluoromethyl)benzene as internal standard. 
Table 3 The palladium-catalyzed direct arylation of substituted thiazoles with aryl bromides $^{\mathrm{a}}$
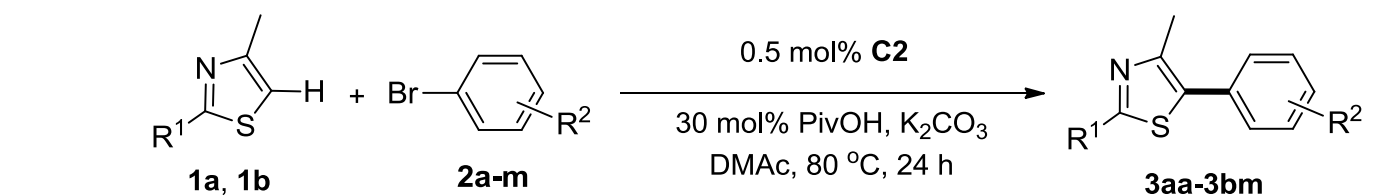

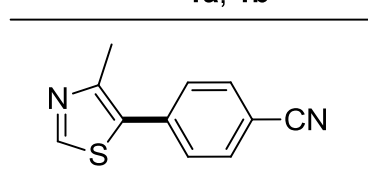

3aa: $92 \%$

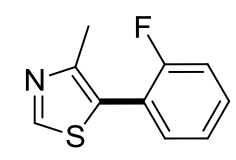

3ae: $68 \%$

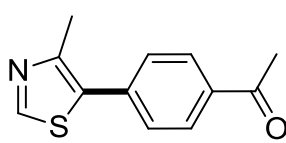

3ai: $88 \%$

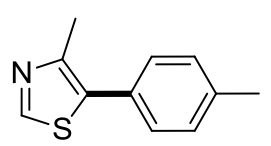

3am: $51 \%$

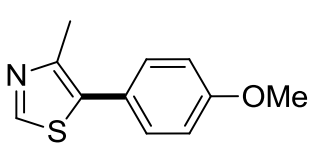

3aq: $39 \%$

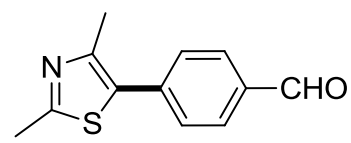

3bc: $96 \%$

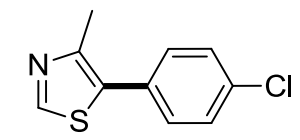

3ab: $86 \%$

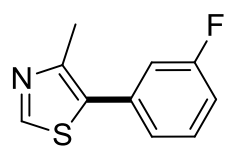

3af: $82 \%$

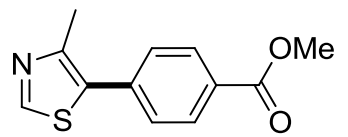

3aj: $90 \%$



3an: $62 \%$

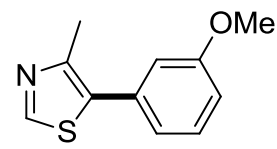

3ar: $55 \%$

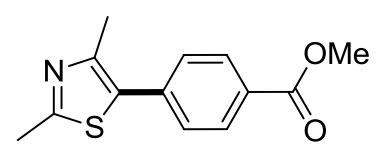

3bj: $97 \%$

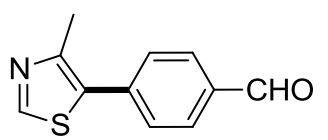

3ac: $98 \%$



3ag: $74 \%$

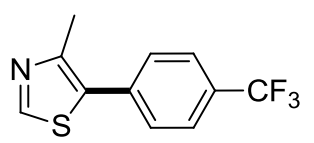

3ak: $93 \%$



3ao: $31 \%$<smiles>Cc1nc(C)c(-c2ccc(C#N)cc2)s1</smiles>

3ba: $98 \%$



3bl: $79 \%$ 3aa-3bm



3ad: $88 \%$


3al: $68 \%$



3ap: $92 \%$<smiles>Cc1nc(C)c(-c2ccc(Cl)cc2)s1</smiles>

3bb: $93 \%$

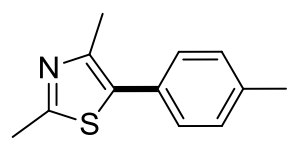

3bm: $68 \%$

${ }^{\mathrm{a} C}$ Conditions: thiazole $(1.0 \mathrm{mmol}), 4$-bromobenzonitrile $(1.0 \mathrm{mmol}), \mathbf{C 2}(0.5 \mathrm{~mol} \%)$, PivOH $(0.3 \mathrm{mmol})$, base $(1.5 \mathrm{mmol})$, solvent $(3 \mathrm{~mL}), 80{ }^{\circ} \mathrm{C}, 24 \mathrm{~h}$, isolated yields. 
Table 4 The direct arylation of substituted thiazoles with aryl bromides at low palladium loading $^{\mathrm{a}}$

\begin{tabular}{|c|c|c|c|c|c|c|}
\hline & & & DMAc, & ${ }^{\circ} \mathrm{C}, 24 \mathrm{~h}$ & $3 a a$ & \\
\hline Entry & Thiazole & $\mathrm{Ar}-\mathrm{Br}$ & & Product & $\operatorname{Pd}(\mathrm{mol} \%)$ & $\begin{array}{c}\text { Yield } \\
(\%)\end{array}$ \\
\hline 1 & & $\mathrm{Br}$ & $\mathrm{CN}$ & 3aa & $\begin{array}{c}0.1 \\
0.05\end{array}$ & $\begin{array}{l}90 \\
71\end{array}$ \\
\hline 2 & & & & 3ab & $\begin{array}{c}0.1 \\
0.05\end{array}$ & $\begin{array}{l}94 \\
82\end{array}$ \\
\hline 3 & & & $\mathrm{CHO}$ & $3 \mathbf{a c}$ & $\begin{array}{c}0.1 \\
0.05\end{array}$ & $\begin{array}{l}96 \\
82\end{array}$ \\
\hline 4 & & & & $3 \mathbf{a e}$ & $\begin{array}{c}0.1 \\
0.05\end{array}$ & $\begin{array}{l}76 \\
69\end{array}$ \\
\hline 5 & & & $\mathrm{CF}_{3}$ & 3ak & $\begin{array}{c}0.1 \\
0.05\end{array}$ & $\begin{array}{l}94 \\
69\end{array}$ \\
\hline 6 & & $\mathrm{Br}$ & & 3am & 0.1 & 63 \\
\hline 7 & & & & 3an & 0.1 & 67 \\
\hline 8 & & & & 3ap & $\begin{array}{c}0.1 \\
0.05\end{array}$ & $\begin{array}{l}89 \\
71\end{array}$ \\
\hline 9 & & $B_{r}$ & -OMe & 3aq & 0.1 & 55 \\
\hline 10 & & & & $3 b b$ & $\begin{array}{c}0.1 \\
0.05\end{array}$ & $\begin{array}{l}91 \\
83\end{array}$ \\
\hline 11 & & & $\mathrm{CHO}$ & $3 b c$ & $\begin{array}{c}0.1 \\
0.05\end{array}$ & $\begin{array}{l}97 \\
78\end{array}$ \\
\hline
\end{tabular}




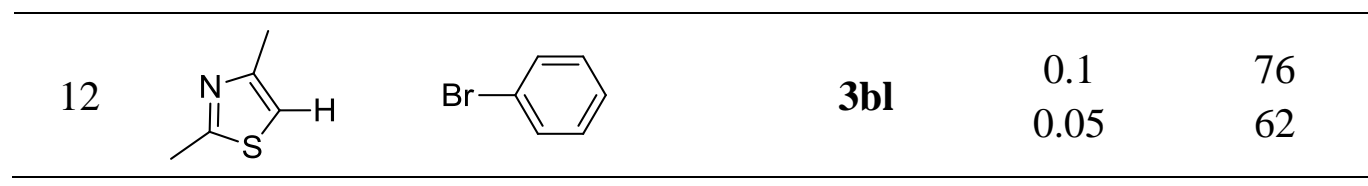

${ }^{\mathrm{a} C}$ Conditions: thiazole $(1.0 \mathrm{mmol})$, aryl bromide $(1.0 \mathrm{mmol}), \mathbf{C 2}(0.1-0.05 \mathrm{~mol} \%)$, PivOH $(0.3 \mathrm{mmol})$, base $(1.5 \mathrm{mmol})$, solvent $(3 \mathrm{~mL}), 100{ }^{\circ} \mathrm{C}, 24 \mathrm{~h}$, isolated yields. 
Scheme 1 Structure of the aniline palladium complexes

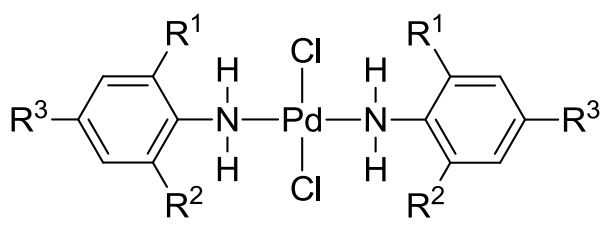

C1: $\mathrm{R}^{1}=i \operatorname{Pr}, \mathrm{R}^{2}=i \operatorname{Pr}, \mathrm{R}^{3}=\mathrm{H}$;

C2: $\mathrm{R}^{1}=\mathrm{Me}, \mathrm{R}^{2}=\mathrm{CHPh}_{2}, \mathrm{R}^{3}=\mathrm{Me}$;

C3: $\mathrm{R}^{1}=\mathrm{CHPh}_{2}, \mathrm{R}^{2}=\mathrm{CHPh}_{2}, \mathrm{R}^{3}=\mathrm{Me}$;

C4: $\mathrm{R}^{1}=\mathrm{CHPh}_{2}, \mathrm{R}^{2}=\mathrm{CHPh}_{2}, \mathrm{R}^{3}=\mathrm{OMe}$;

C5: $\mathrm{R}^{1}=\mathrm{CHPh}_{2}, \mathrm{R}^{2}=\mathrm{CHPh}_{2}, \mathrm{R}^{3}=\mathrm{Cl}$; 
Scheme 2 Proposed catalytic cycle for direct arylation of thiazoles with aryl bromides

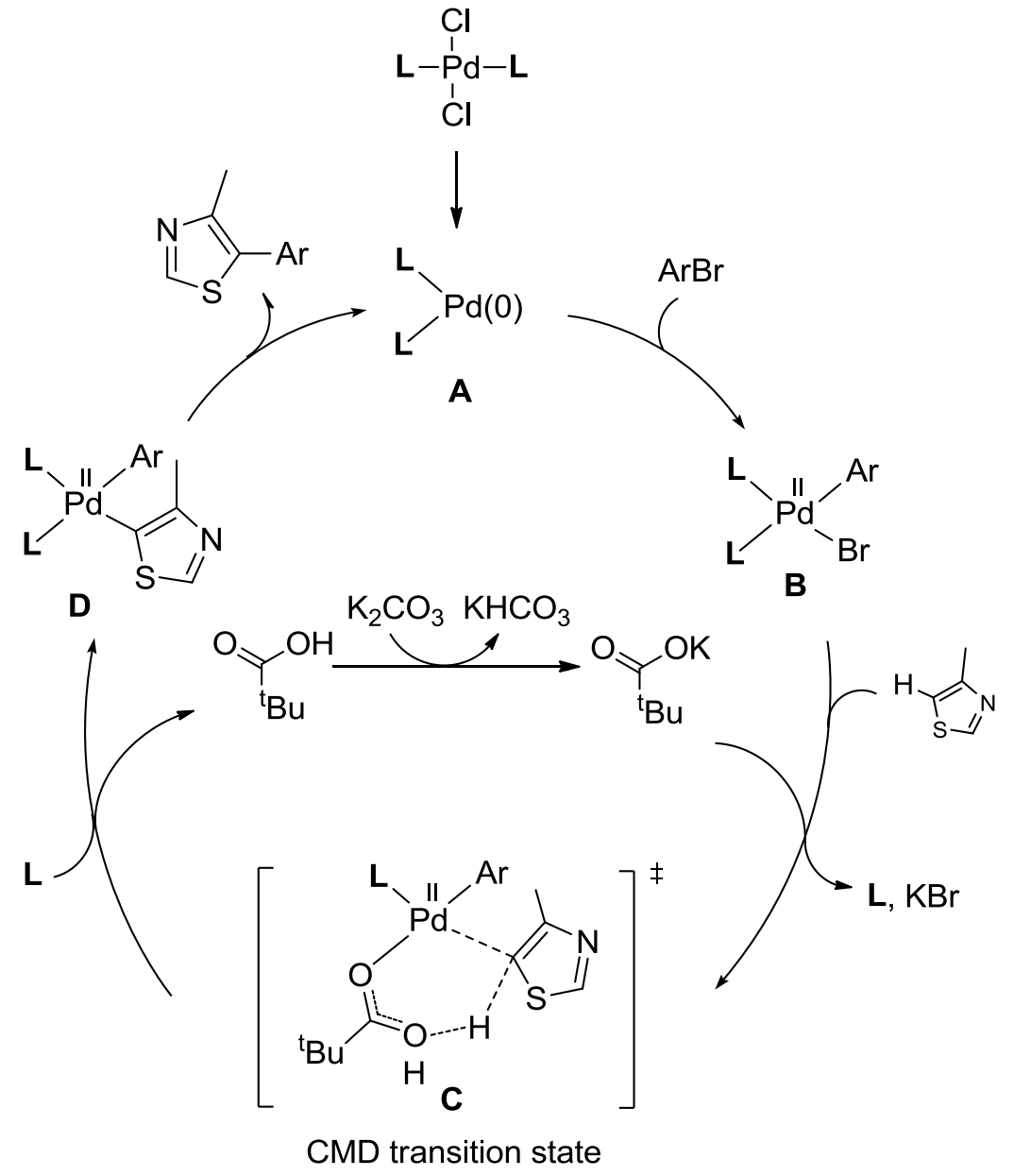




\section{Tables}

Table 1 Screening of palladium complexes on direct arylation reaction of 4-methylthiazole with 4-bromobenzonitrile ${ }^{\mathrm{a}}$

Table 2 Screening of reaction conditions for the direct arylation reaction of 4-methylthiazole with 4-bromobenzonitrile ${ }^{\mathrm{a}}$

Table 3 The palladium-catalyzed direct arylation of substituted thiazoles with aryl bromides $^{\mathrm{a}}$

Table 4 The direct arylation of substituted thiazoles with aryl bromides at low palladium loading ${ }^{\mathrm{a}}$ 
Table 1 Screening of palladium complexes on direct arylation reaction of 4-methylthiazole with 4-bromobenzonitrile ${ }^{\mathrm{a}}$<smiles>Cc1ncsc1C</smiles>

1a

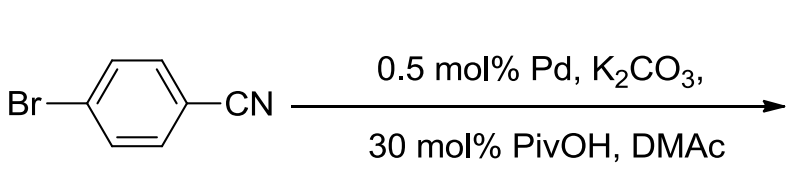

2a



3aa

\begin{tabular}{cccc}
\hline Entry & Catalyst & $\mathrm{T}\left({ }^{\circ} \mathrm{C}\right)$ & Cov. $(\%)^{\mathrm{b}}$ \\
1 & $\mathbf{2 C y}_{\mathbf{2}} \mathbf{N H} / \mathbf{P d C l}_{\mathbf{2}}$ & 80 & 86 \\
2 & $\mathbf{C 1}$ & 80 & 85 \\
3 & $\mathbf{C 2}$ & 80 & $96(92)^{\mathrm{c}}$ \\
4 & $\mathbf{C 3}$ & 80 & 90 \\
5 & $\mathbf{C 4}$ & 80 & 91 \\
6 & $\mathbf{C 5}$ & 80 & 89 \\
\hline
\end{tabular}

${ }^{a}$ Reaction conditions: 4-methylthiazole $(1 \mathrm{mmol})$, 4-bromobenzonitrile (1 mmol), palladium source $(0.5 \mathrm{~mol} \%)$, PivOH $(0.3 \mathrm{mmol})$, base $(1.5 \mathrm{mmol})$, DMAc (3 mL) under aerobic environment for $24 \mathrm{~h} .{ }^{\mathrm{b}} \mathrm{GC}$ yield using the (trifluoromethyl)benzene as internal standard. ${ }^{c}$ isolated yields in parentheses. 
Table 2 Screening of reaction conditions for the direct arylation reaction of 4-methylthiazole with 4-bromobenzonitrile ${ }^{\mathrm{a}}$

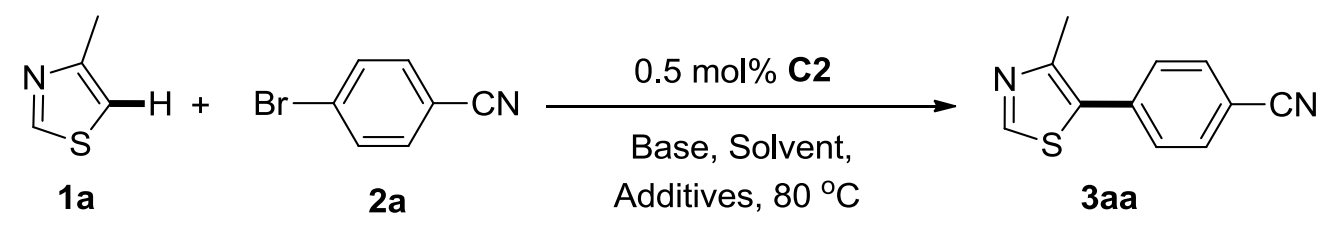

\begin{tabular}{|c|c|c|c|c|}
\hline Entry & Base & Solvent & Additive & Cov. $(\%)^{\mathrm{b}}$ \\
\hline 1 & $\mathrm{~K}_{2} \mathrm{CO}_{3}$ & DMAc & $\mathrm{PivOH}$ & 96 \\
\hline 2 & $\mathrm{Na}_{2} \mathrm{CO}_{3}$ & DMAc & $\mathrm{PivOH}$ & 47 \\
\hline 3 & $\mathrm{NaHCO}_{3}$ & DMAc & $\mathrm{PivOH}$ & 47 \\
\hline 4 & $\mathrm{C}_{6} \mathrm{H}_{5} \mathrm{CO}_{2} \mathrm{Na}$ & DMAc & $\mathrm{PivOH}$ & 29 \\
\hline 5 & $\mathrm{~K}_{3} \mathrm{PO}_{4}$ & DMAc & $\mathrm{PivOH}$ & 71 \\
\hline 6 & KOAc & DMAc & $\mathrm{PivOH}$ & 90 \\
\hline 7 & $\mathrm{NaOH}$ & DMAc & $\mathrm{PivOH}$ & 6 \\
\hline 8 & $\mathrm{NaOAc}$ & DMAc & $\mathrm{PivOH}$ & 30 \\
\hline 9 & $\mathrm{Cs}_{2} \mathrm{CO}_{3}$ & DMAc & $\mathrm{PivOH}$ & 5 \\
\hline 10 & $\mathrm{KOH}$ & DMAc & $\mathrm{PivOH}$ & NR \\
\hline 11 & $\mathrm{KO}^{\mathrm{t}} \mathrm{Bu}$ & DMAc & $\mathrm{PivOH}$ & NR \\
\hline 12 & $\mathrm{NaO}^{t} \mathrm{Bu}$ & DMAc & $\mathrm{PivOH}$ & NR \\
\hline 13 & $\mathrm{~K}_{2} \mathrm{CO}_{3}$ & DMF & $\mathrm{PivOH}$ & 84 \\
\hline 14 & $\mathrm{~K}_{2} \mathrm{CO}_{3}$ & Dioxane & $\mathrm{PivOH}$ & 70 \\
\hline 15 & $\mathrm{~K}_{2} \mathrm{CO}_{3}$ & Toluene & $\mathrm{PivOH}$ & 50 \\
\hline 16 & $\mathrm{~K}_{2} \mathrm{CO}_{3}$ & Xylene & $\mathrm{PivOH}$ & 39 \\
\hline 17 & $\mathrm{~K}_{2} \mathrm{CO}_{3}$ & NMP & $\mathrm{PivOH}$ & 62 \\
\hline 18 & $\mathrm{~K}_{2} \mathrm{CO}_{3}$ & DMSO & $\mathrm{PivOH}$ & NR \\
\hline 19 & $\mathrm{~K}_{2} \mathrm{CO}_{3}$ & $\mathrm{EtOH}$ & $\mathrm{PivOH}$ & NR \\
\hline 20 & $\mathrm{~K}_{2} \mathrm{CO}_{3}$ & DMAc & - & 9 \\
\hline 21 & $\mathrm{~K}_{2} \mathrm{CO}_{3}$ & DMAc & HOAc & 67 \\
\hline 22 & $\mathrm{~K}_{2} \mathrm{CO}_{3}$ & DMAc & $\mathrm{C}_{2} \mathrm{H}_{5} \mathrm{COOH}$ & 66 \\
\hline 23 & $\mathrm{~K}_{2} \mathrm{CO}_{3}$ & DMAc & $\mathrm{CF}_{3} \mathrm{COOH}$ & 14 \\
\hline 24 & $\mathrm{~K}_{2} \mathrm{CO}_{3}$ & DMAc & Lactic acid & 15 \\
\hline 25 & $\mathrm{~K}_{2} \mathrm{CO}_{3}$ & DMAc & $\mathrm{CH}_{2}(\mathrm{COOH})_{2}$ & 19 \\
\hline 26 & $\mathrm{~K}_{2} \mathrm{CO}_{3}$ & DMAc & o-Phthalic Acid & 11 \\
\hline 27 & $\mathrm{~K}_{2} \mathrm{CO}_{3}$ & DMAc & Benzoic acid & 32 \\
\hline
\end{tabular}

${ }^{\mathrm{a}}$ Conditions: 1a $(1.0 \mathrm{mmol})$, 4-bromobenzonitrile $(1.0 \mathrm{mmol}), \mathbf{C 2}(0.5 \mathrm{~mol} \%)$, Additives $(0.3 \mathrm{mmol})$, base $(1.5 \mathrm{mmol})$, solvent $(3 \mathrm{~mL}), 80{ }^{\circ} \mathrm{C}, 24 \mathrm{~h} .{ }^{\mathrm{b}} \mathrm{GC}$ yield using the (trifluoromethyl)benzene as internal standard. 
Table 3 The palladium-catalyzed direct arylation of substituted thiazoles with aryl bromides $^{\mathrm{a}}$
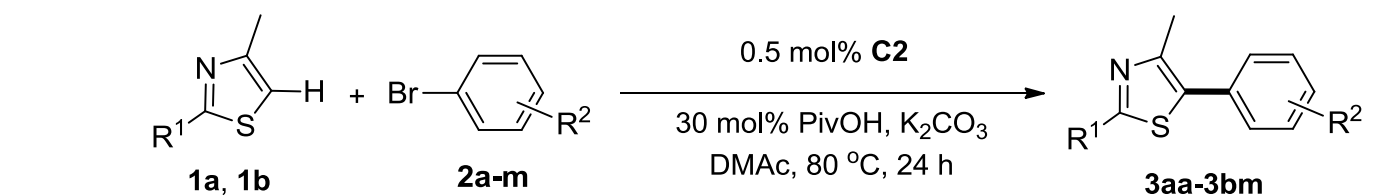



3aa: $92 \%$

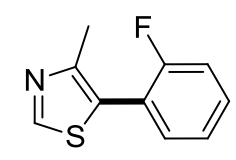

3ae: $68 \%$

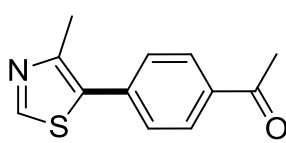

3ai: $88 \%$

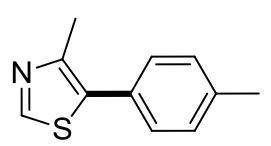

3am: $51 \%$

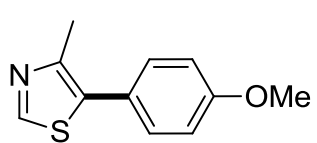

3aq: $39 \%$

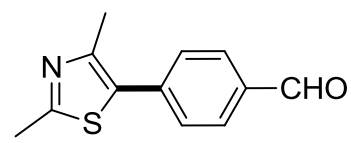

3bc: $96 \%$

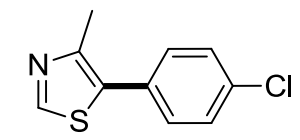

3ab: $86 \%$

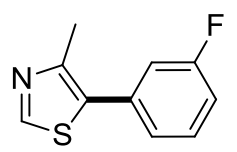

3af: $82 \%$

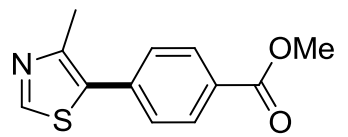

3aj: $90 \%$

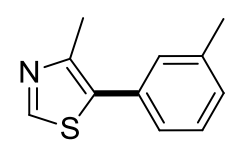

3an: $62 \%$

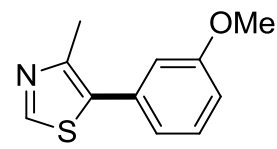

3ar: $55 \%$

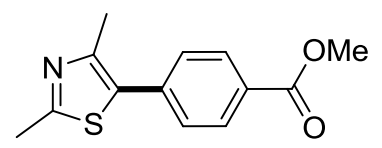

3bj: $97 \%$

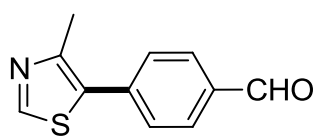

3ac: $98 \%$



3ag: $74 \%$

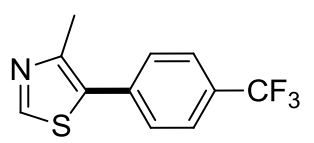

3ak: $93 \%$

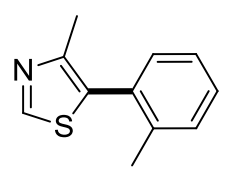

3ao: $31 \%$<smiles>Cc1nc(C)c(-c2ccc(C#N)cc2)s1</smiles>

3ba: $98 \%$



3bl: $79 \%$ 3aa-3bm

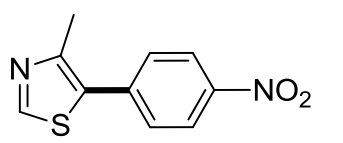

3ad: $88 \%$
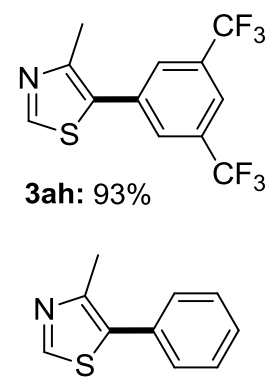

3al: $68 \%$

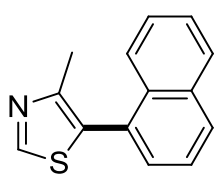

3ap: $92 \%$<smiles>Cc1nc(C)c(-c2ccc(Cl)cc2)s1</smiles>

3bb: $93 \%$

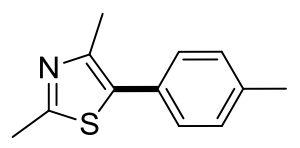

3bm: $68 \%$

${ }^{\mathrm{a} C}$ Conditions: thiazole $(1.0 \mathrm{mmol}), 4$-bromobenzonitrile $(1.0 \mathrm{mmol}), \mathbf{C 2}(0.5 \mathrm{~mol} \%)$, PivOH $(0.3 \mathrm{mmol})$, base $(1.5 \mathrm{mmol})$, solvent $(3 \mathrm{~mL}), 80{ }^{\circ} \mathrm{C}, 24 \mathrm{~h}$, isolated yields. 
Table 4 The direct arylation of substituted thiazoles with aryl bromides at low palladium loading $^{\mathrm{a}}$

\begin{tabular}{|c|c|c|c|c|c|c|}
\hline & & & DMAc, & ${ }^{\circ} \mathrm{C}, 24 \mathrm{~h}$ & $3 a a$ & \\
\hline Entry & Thiazole & $\mathrm{Ar}-\mathrm{Br}$ & & Product & $\operatorname{Pd}(\mathrm{mol} \%)$ & $\begin{array}{c}\text { Yield } \\
(\%)\end{array}$ \\
\hline 1 & & $\mathrm{Br}$ & $\mathrm{CN}$ & 3aa & $\begin{array}{c}0.1 \\
0.05\end{array}$ & $\begin{array}{l}90 \\
71\end{array}$ \\
\hline 2 & & & & 3ab & $\begin{array}{c}0.1 \\
0.05\end{array}$ & $\begin{array}{l}94 \\
82\end{array}$ \\
\hline 3 & & & $\mathrm{CHO}$ & $3 \mathbf{a c}$ & $\begin{array}{c}0.1 \\
0.05\end{array}$ & $\begin{array}{l}96 \\
82\end{array}$ \\
\hline 4 & & & & $3 \mathbf{a e}$ & $\begin{array}{c}0.1 \\
0.05\end{array}$ & $\begin{array}{l}76 \\
69\end{array}$ \\
\hline 5 & & & $\mathrm{CF}_{3}$ & 3ak & $\begin{array}{c}0.1 \\
0.05\end{array}$ & $\begin{array}{l}94 \\
69\end{array}$ \\
\hline 6 & & $\mathrm{Br}$ & & 3am & 0.1 & 63 \\
\hline 7 & & & & 3an & 0.1 & 67 \\
\hline 8 & & & & 3ap & $\begin{array}{c}0.1 \\
0.05\end{array}$ & $\begin{array}{l}89 \\
71\end{array}$ \\
\hline 9 & & $B_{r}$ & -OMe & 3aq & 0.1 & 55 \\
\hline 10 & & & & $3 b b$ & $\begin{array}{c}0.1 \\
0.05\end{array}$ & $\begin{array}{l}91 \\
83\end{array}$ \\
\hline 11 & & & $\mathrm{CHO}$ & $3 b c$ & $\begin{array}{c}0.1 \\
0.05\end{array}$ & $\begin{array}{l}97 \\
78\end{array}$ \\
\hline
\end{tabular}




12

${ }^{\mathrm{a} C}$ Conditions: thiazole $(1.0 \mathrm{mmol})$, aryl bromide $(1.0 \mathrm{mmol}), \mathbf{C 2}(0.1-0.05 \mathrm{~mol} \%)$, PivOH $(0.3 \mathrm{mmol})$, base $(1.5 \mathrm{mmol})$, solvent $(3 \mathrm{~mL}), 100{ }^{\circ} \mathrm{C}, 24 \mathrm{~h}$, isolated yields. 


\section{Graphics}

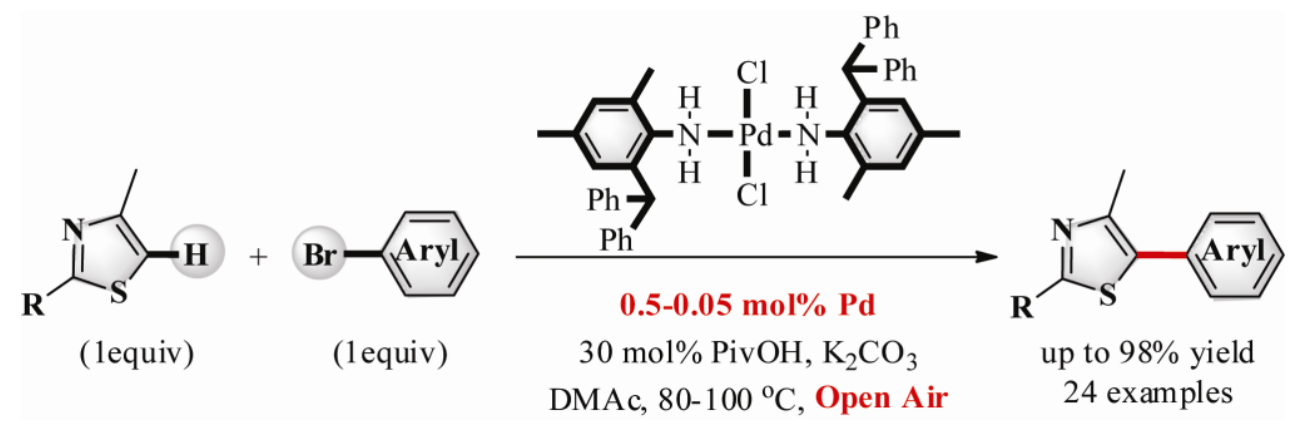

A series of bulky aniline palladium complexes were synthesized and characterized, which exhibited significantly activities for direct arylation of thiazoles with aryl bromides. 